\title{
The extracellular matrix gene, Svep1, orchestrates airway patterning and the transition from lung branching morphogenesis to alveolar maturation in the mouse
}

\author{
Foxworth $\mathrm{N}^{1^{*}}$, Wells $\mathrm{J}^{2^{*}}$, Ocaña-Lopez $\mathrm{S}^{1}$, Muller $\mathrm{S}^{1,3,4}$, Denegre $\mathrm{J}^{2}$, Palmer $\mathrm{K}^{2}$, McGee $\mathrm{T}^{2}$, \\ Memishian $\mathrm{W}^{2}$, Murray $\mathrm{SA}^{2}$, Donahoe $\mathrm{PK}^{1,3}$, Bult $\mathrm{CJ}^{2,+}$, and Loscertales $\mathrm{M}^{1,3,+}$ \\ ${ }^{1}$ Pediatric Surgical Research Laboratories, Massachusetts General Hospital, Boston, MA \\ 02114 \\ ${ }^{2}$ The Jackson Laboratory, Bar Harbor, ME 04609 \\ ${ }^{3}$ Harvard Medical School, Boston, MA 02114 \\ ${ }^{4}$ Broad Institute of MIT, Cambridge, MA 02142 \\ "Equal Contributions \\ ${ }^{+}$Co-senior authors
}

Correspondence: Maria Loscertales, mloscertales@mgh.harvard.edu; Carol J. Bult, carol.bult@jax.org

Author Contributions: ML designed and led the execution, data analysis, and interpretation for lung phenotyping, imaging, RNA Seq, and lung explant experiments with contributions from NF and SOL. SM contributed to the analysis of the RNA Seq data. Svep1 mutants were generated by the Knockout Mouse Project center at The Jackson Laboratory under the direction of SAM. JD and KP performed microCT on Svep1 mutants and maintained the initial colonies of Svep ${ }^{\text {\% }}$ animals with oversight from SAM. JW, WM, TG performed animal husbandry for timed matings to generate embryos for lung phenotyping and performed the scRNA Seq experiment. TM, WM and $C J B$ analyzed the scRNA Seq data. ML and CJB wrote the manuscript in consultation with PKD, JW, SM, TM, and WM.

Competing Interest Statement: The authors have declared no competing interest.

Data availability: Transcriptomics data from this study will be submitted to a public data archive upon acceptance in a peer-reviewed journal.

Keywords: Svep1, lung development, lung branching, alveolar differentiation 


\section{Abstract}

Proper lung development and function requires two independent but interrelated processes: branching morphogenesis to form the airway tree, and alveolar cell differentiation for peripheral gas exchange. The disruption of either branching or differentiation results in severe respiratory deficiencies and often in neonatal death. The molecular mechanisms that control branching patterns and the transition to alveolar differentiation are not completely understood. Here we report on the in vitro and in vivo characterization of the lungs of mouse embryos lacking a functional Svep1 gene. Our data demonstrate that the SVEP1 extracellular matrix protein is critical for airway patterning and for the process of transitioning from branching to alveolar maturation. Svep1 $1^{-/}$embryos on a C57BL/6J genetic background are characterized by hypoplastic lungs and a disorganized increase in distal airway tips which disrupts airway architecture and lobe shape. The lungs of Svep 1 knockout embryos also have defects in alveolar differentiation. In vitro lung explant experiments demonstrated that SVEP1 normally inhibits branching morphogenesis and that treatment with a SVEP1 peptide can rescue the branching defects observed in Svep1 knockouts. Our findings reveal for the first time that Svep1 is essential for constructing the basic airway architecture and for the transition from lung branching to alveolar differentiation. Our results suggest therapeutic strategies to enhance lung development in patients with life-threatening respiratory disorders such as the lung hypoplasia and prematurity observed in neonates with congenital diaphragmatic hernia (CDH).

\section{Introduction}

The mammalian lung consists of conductive airways (bronchi and bronchioles) and terminal alveoli which function in peripheral gas exchange. Two developmental processes underlie the lung's basic architecture: branching morphogenesis to build the airway tree structure, and differentiation to generate specialized cells. Cellular differentiation to form specialized cells in different regions of the lung occurs predominantly after the formation of the tracheobronchial tree ${ }^{1}$. For alveolar cell differentiation to occur, the molecular genetic signaling that promotes branching must cease as lung branching antagonizes differentiation ${ }^{2}$.

In the laboratory mouse, branching morphogenesis starts soon after the formation of the lung buds from the ventral foregut endoderm at embryonic day 9.5 (E9.5). During the pseudoglandular stage (E12.5 - E16.5), epithelial progenitors at the bud tips undergo an iterative process of budding and bifurcations into the surrounding mesenchyme. During the canalicular (E16.5-17.5) stage the respiratory tree is expanded in diameter and length. The vasculature develops along the airway and terminal airway tips widen to form saccules. During the saccular stage (E17.5-P5) the alveolar epithelium differentiates into alveoli-cuboidal cells (type II pneumocytes) responsible for surfactant protein C (SPC) production and the thin gasexchanging cells (type I pneumocytes). Primitive septa arise at this stage. Secondary septation and alveolar maturation occur during postnatal development ${ }^{3}$.

The airway tree geometry of the lung is generated by three local branching modes: domain, planar, and orthogonal ${ }^{4}$. During domain branching, new branches grow perpendicularly around 
the circumference of the parent branch and generate the initial scaffold of each lobe which, in turn, determines the overall shape of the lung. Planar bifurcation occurs at the tips of the airway epithelium and generates branches in the same plane resulting in the thin edges of the lung lobes. Orthogonal bifurcation generates branches perpendicular to prior branches thus filling in the interior. Trifurcations are rare and appear principally at the lung surface ${ }^{5 ; 6}$. As branching proceeds, proximal-distal patterning is established, and cellular specification follows ${ }^{7-9}$. Thus, bud tip progenitors persist and proliferate, then undergo differentiation into type I and type II pneumocytes ${ }^{8}$, and the cells of the primal airway stalk differentiate into multiple proximal cell types ${ }^{10-13}$.

Mesenchymal-epithelial interactions are essential for branching morphogenesis and several signaling pathways, including the FGF pathway, play central roles in lung morphogenesis ${ }^{14}$. Fibroblast growth factor 10 (FGF10) secreted by mesenchymal cells acts through the FGFR2 receptor to maintain a permissive environment for branching morphogenesis. FGF10 maintains distal epithelial progenitor cells (SOX9 ${ }^{+}$cells) in an undifferentiated state ${ }^{15-26}$. FGF10 signaling plays a pivotal role in alveolar and mesenchymal differentiation as $\mathrm{FGF} 10^{+}$cells give rise to bronchial airway smooth muscle cells during early branching ${ }^{27-30}$ The extra cellular matrix (ECM) also plays important roles in lung morphogenesis, including epithelial branching and alveolarization ${ }^{31-36}$. The ECM also contributes to organ shape ${ }^{37}$.

SVEP1 (Sushi, Von Willebrand Factor Type A, EGF, and Pentratix Domains-Containing 1; HGNC:15985), also called Polydom, is an extracellular matrix (ECM) protein which is ubiquitously expressed in adult mice and plays roles in cell adhesion ${ }^{38}$, epidermal differentiation ${ }^{39}$, and lymphatic vessel formation and remodeling ${ }^{40 ; 41}$. According to the Mouse Genome Informatics (MGI) database ${ }^{42}$, Svep1(MGI:1928849) homozygous mutant mice display "complete preweaning lethality, edema, abnormal skin coloration, thick epidermis, acanthosis, tail/limb abnormalities, and defects in lymphatic vascular development and valve formation" $39 ; 41$; $43 ; 44$.

We investigated the role of Svep1 in branching morphogenesis and alveolar cellular differentiation by in vivo and in vitro characterization of the lungs of mice homozygous for the

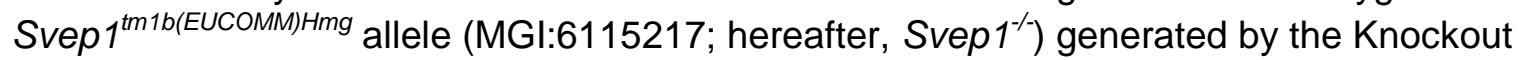
Mouse Phenotyping Program (KOMP2) program at The Jackson Laboratory ${ }^{44}$. We determined that the loss of Svep1 disrupts the basic lung branching program resulting in an irregular lung lobe shape due to an aberrant increase in distal airway tips. Alveolar and smooth muscle differentiation is also disrupted in the lungs of Svep $1^{-1-}$ embryos. We demonstrated that SVEP1 acts as a negative regulator of branching and that branching abnormalities, but not airway smooth muscle differentiation, are independent of FGF signaling. As the loss of Svep1 disrupts alveolar differentiation, SVEP1 peptides or inhibition of SVEP1 downstream targets such as SOX9 and members of the FGF signaling pathway are potential candidates for therapeutic interventions for conditions in which lung prematurity is a major contributor to mortality. 


\section{Methods}

Svep1 mutant mice.Svep1 mutant mice (B6N(Cg)-Svep $\left.1^{\text {tm } 1 b(E U C O M M) H m g u} / \mathrm{J}\right)$ were generated as part of the Knockout Mouse Phenotyping Program (KOMP2) by inserting the L1L2_Bact_P cassette upstream of exon 8 of Svep1. This cassette contains a flippase recombinase target (FRT) site followed by a lacZ sequence and a loxP site. The first loxP site is followed by a neomycin resistance gene, a second FRT site, and a second loxP site. A third loxP site is inserted downstream of exon 8 . The construct was introduced into embryonic cells and embryonic stem cell clone HEPD0747_6_B06 was injected into B6(Cg)-Tyr $\mathrm{r}^{\mathrm{c} 2 \mathrm{~J}} / \mathrm{J}$ blastocysts. Resulting chimeric males were bred to $\mathrm{C} 57 \mathrm{BL} / 6 \mathrm{NJ}$ female mice to generate heterozygous tm1a (i.e., knockout first) animals ${ }^{45}$. No homozygous mice were recovered at weaning from heterozygous intercrosses. Heterozygous tm1a mice were then bred to B6N.Cg-Tg (Sox2cre) $1 \mathrm{Amc} / \mathrm{J}$ mice to remove the floxed neomycin sequence and exon 8 of Svep1. Resulting offspring were bred to $\mathrm{C} 57 \mathrm{BL} / 6 \mathrm{NJ}$ mice to remove the cre-expressing transgene.

Mice and embryos were genotyped by PCR using the primers shown below. The wild type (WT) PCR product is 389 base pairs (bp) in length and the mutant (MUT) product is 540 bp. DNA was isolated from tissues obtained by either ear notching or tail tipping using the Hot Shot method ${ }^{46}$. Each PCR reaction consisted of $1 \mu \mathrm{g}$ of DNA, $1 \mu \mathrm{g}$ of each genotyping primer, $4 \mu \mathrm{l}$ of $5 \mathrm{M}$ betaine, $4 \mu \mathrm{l}$ of $5 \mathrm{X}$ Phusion buffer, $2 \mu \mathrm{l}$ of dNTP mix (10 mM each dNTP;) and $0.2 \mu \mathrm{l}$ of Phusion DNA polymerase. Reactions were amplified by 25 cycles of PCR with an annealing temperature of $60^{\circ} \mathrm{C}$ and visualized by electrophoresis on a $2 \%$ agarose gel.

$\begin{array}{cc}\text { primer name } & \text { primer sequence } \\ \text { Svep1 WT F } & \text { 5'-AGCTTTTCCACTCTAGCAAGC-3' } \\ \text { Svep1 WT R } & \text { 5'-CATGCAGGTCCTTTCATCCT-3' } \\ \text { Svep1 MUT F } & \text { 5'-CGGTCGCTACCATTACCAGT-3' } \\ \text { Svep1 MUT R } & \text { 5'-TCCATCCTTCAGATTTGGTCA-3' }\end{array}$

Histology. Mouse embryos were euthanized by decapitation at E14.5 E16.5, or E18.5. Tails were collected for genotyping and whole lungs were extracted and fixed in $4 \%$ paraformaldehyde (PFA) and embedded in paraffin. Paraffin tissue blocks were sectioned (5 $\mu \mathrm{m})$ and stained for Hematoxylin and Eosin (H\&E) following standard procedures. Images were acquired using a Nikon80i microscope

For Toluidine Blue Staining, mouse embryos were euthanized by decapitation at E18.5. Tails were collected for genotyping and lungs were fixed in a combination of $2 \%$ paraformaldehyde (PFA)/2\% glutaraldehyde in $0.1 \mathrm{M}$ cacodylate buffer for $24 \mathrm{hr}$ at $4^{0} \mathrm{C}$. Lungs were post-fixed in $2 \%$ aqueous osmium tetroxide for $2 \mathrm{hr}$ at room temperature, rinsed in $1 \mathrm{X}$ phosphate buffered saline (PBS), then dehydrated with a series of ethanol, from $40 \%$ through $100 \%$. For each ethanol stage, lungs were incubated for 15 minutes at room temperature. The final $100 \%$ ethanol stage was repeated three times. Dehydrated embryos were infiltrated with Embed 812 resin (Electron Microscopy Sciences, Hatfield, PA) and embedded in the same resin. The blocks with embedded embryos were cured at $60^{\circ} \mathrm{C}$ for $24 \mathrm{hr}$. One-micron thick 
sections were cut using a diamond knife and baked onto glass slides. Slides were covered in $0.5 \%$ aqueous toluidine blue for 30 seconds, rinsed in running water, and then allowed to air dry before a coverslip was placed on the slide.

In situ hybridization (ISH). For whole mount in situ hybridization of Svep1, a 725bp segment of the Svep1 transcript (NM_153366.4; 746-11179) was PCR amplified (PCR Master Mix, Promega) with one pair of exon-exon boundary overlapping primers (5' TGTGGTCCTCCAAGTCACGTA 3'; 5' CCAGCAGACAGCAGAGTATGT 3') designed using

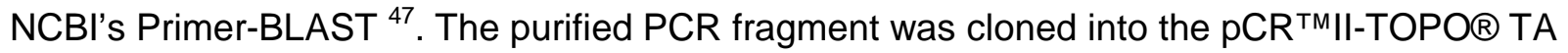
vector (TOPO® TA Cloning® Kit, Dual Promoter, ThermoFisher), and transformed into One Shot ${ }^{\circledR}$ TOP10 Chemically Competent Cells, ThermoFisher). Transformed colonies on agar plates were selected by ampicillin resistance following $24 \mathrm{hr}$ at $37^{\circ} \mathrm{C}$. The orientation of the insert after linearization of the vector with Spel restriction digestion (New England Biolabs, Inc.) was determined by Sanger sequencing. Sense and anti-sense Digoxigenin-11-UTP labeled probes (DIG RNA Labeling Mix, Sigma-Aldrich) were synthesized with SP6 and T7 RNA polymerases, respectively. In situ hybridization was performed using techniques minimally altered from those published by Riddle et al. ${ }^{48}$ and developed using BM purple AP substrate (Roche) per the manufacturer's instructions.

$5 \mu \mathrm{m}$ thick sections of paraffin embedded lung tissues were used for ISH of Fgf10 (NM_446371, 862-1978 nt, Advance Diagnostic), Fgf9 (NM_013518.4, 27-1198 nt), Fgfr2 (NM_010207, 2-1677nt), and Svep1 (NM_022814.2, 2879 -3726 nt) using RNAscope 2.0 Red Detection Kit (Advanced Cell Diagnostic) according to the manufacturer's instructions.

Immunohistochemistry. For the quantification of lung distal epithelial tips, fixed whole lungs were washed with PBS and permeabilized in $0.5 \%$ Triton-X, 5\% BSA in PBS for $1 \mathrm{hr}$ at room temp. SOX9 primary antibody diluted in 1X PBS was incubated overnight at room temperature. Samples were washed 3 times for 15 min in PBS. An Alexa fluor (Life Technology, USA) secondary antibody diluted in 1X PBS was incubated for $2 \mathrm{hr}$ at room temperature. Samples were mounted with DAPI mounting medium. Samples were washed again before fixation with 4\% PFA in PBS for 2-4 hr. Quantification was performed using ImageJ ${ }^{49}$ and statistical significance was determined using a two-tailed t-test with significance at $p<0.05$.

To evaluate branching patterns, fixed whole lungs from E13.5 embryos were dehydrated in a methanol series, washed with $20 \%$ DMSO in methanol, and then re-hydrated with a methanol series. Lungs were then permeabilized with $0.2 \%$ Triton-X in PBS, treated with Visikol Penetration Buffer for $2 \mathrm{hr}$, and then stored in Visikol Blocking Buffer overnight at $37^{\circ} \mathrm{C}$. Primary antibodies were diluted in Visikol Antibody Buffer and lungs were incubated overnight at $37^{\circ} \mathrm{C}$ in the antibody mixture. Lungs were washed with Visikol Wash Buffer, then treated with secondary antibodies diluted in Visikol Antibody Buffer overnight at $37^{\circ} \mathrm{C}$. Lungs were washed again with Visikol Wash Buffer, then dehydrated in a methanol series. Finally, the lungs were treated with Visikol HISTO-1 solution for $4 \mathrm{hr}$, followed by Visikol HISTO-2 solution for $4 \mathrm{hr}$. Lungs were mounted on slides using silicone-imaging chambers filled with Visikol HISTO-2 and imaged with a Keyence microscope. Z stack image series were generated for the whole lung 
and used to create a video to trace the lineages of airway branches for orthogonal and planar bifurcation and for quantification of trifurcation and ectopic branching. Statistical significance was determined using a two-tailed t-test with significance at $p<0.05$.

For whole mount lung explant branching immunofluorescence, fixed lungs from E12.5 embryos were placed in PBS overnight at $4^{\circ} \mathrm{C}$, then dehydrated through a PBS/methanol gradient and bleached with $6 \%$ (vol/vol) hydrogen peroxide (H1009, Sigma) in methanol overnight at $4^{\circ} \mathrm{C}$. The following day, samples were rehydrated in a methanol/PBS gradient, then blocked in PBS $+0.3 \%$ Triton X-100 + 5\% normal goat serum for $2 \mathrm{hr}$, followed by addition of primary antibodies into blocking solution and incubated overnight at $4^{\circ} \mathrm{C}$. Samples were then washed 3 times for 15 min with PBS $+0.1 \%$ Triton X-100 + 0.1\% Tween-20, followed by addition of Alexa Fluor antibodies (Life Technology) diluted in PBS $+0.3 \%$ Triton $\mathrm{X}-100$ at $4{ }^{\circ} \mathrm{C}$ overnight. Samples were mounted with DAPI mounting medium. Samples were washed again before fixation with 4\% PFA in PBS for 2-4 hr.

Section Immunohistochemistry (IHC) was performed using standard techniques. Antigen retrieval was achieved by heat treatment in a microwave oven for $20 \mathrm{~min}$ at low power in $0.01 \mathrm{M}$ sodium citrate buffer at $\mathrm{pH} 6$. In tissues imaged using fluorescence, Alexa Fluor secondary antibodies were used (Life Technology). Images were obtained with a Nikon80i or Keyence microscope.

The following antibodies were used for IHC: anti-a-SMA (1:200; rabbit polyclonal; Abcam), anti-a-SMA (1:200; mouse polyclonal; Santa Cruz), anti-prosurfactant protein C (1:400; rabbit polyclonal; Abcam), anti-prosurfactant protein C (1:50; goat polyclonal; Santa Cruz), anti-Ki67 (1:50; rat monoclonal; Invitrogen), anti-CD31 (1:50; rabbit polyclonal; Abcam), anti-PDGFR- $\alpha$ (1:200; rabbit polyclonal; SantaCruz), anti-podoplanin (hamster monoclonal; Abcam), antiSOX9 (rabbit monoclonal; Abcam), anti-E-Cadherin (1:200; mouse monoclonal; Abcam), antiE-Cadherin (1:100; rat monoclonal; Invitrogen), anti-ERG (1:50; rabbit monoclonal; Abcam), anti-FOXJ1 (1:200; mouse monoclonal; Santa Cruz), anti-SOX2 (rat monoclonal; Invitrogen), and Uteroglobin (rat polyclonal; Abcam).

Embryonic lung explant imaging. Lungs from E12.5 embryos were dissected and transferred to a Corning transwell cell culture well plate insert (Millipore Sigma) containing $2 \mathrm{ml}$ of DMEM/F12 medium supplemented with $1 \mathrm{U} / \mathrm{ml}$ penicillin-streptomycin. Lung were incubated in the chamber and time lapse images were recorded every 10 min over $48 \mathrm{hr}$ with a Keyence microscope. Time lapse videos were generated using the Keyence software.

Treatment of lung explant culture and morphometrics. Lung explants from E12.5 Svep $1^{+/+}$and Svep $1^{-/}$embryos were treated for 2 days with a 146 amino acid SVEP1 peptide fragment from the Sushi or Complement Control Protein (CCP) module repeat domain 4 (WGAANRLDYSYDDFLDTVQETATSIGNAKSSRIKRSAPLS DYKIKLIFNITASVPLPDER NDTLEWENQQRLLQTLETITNKLKRTLNKDPMYSFQLASEILIADSNSLETKKASPFCRPGSVLR GRMCVNCPLGTYYNLEHFTCESC) produced in E. coli, as a recombinant protein antigen of 
Svep1 (10 $\mu \mathrm{g} / \mathrm{ml}, \mathrm{NP}$ 0735725.2, region 874-971, Novus Biologicals) or for 2 to 4 days with an inhibitor of FGF signaling (2.5 $\mu \mathrm{M}$ SU5401, Pfizer).

The fold change of epithelial branches in lung explant cultures was determined by counting the starting number of individual branches at $t=0$, then counting new epithelial buds observed emerging from the lung epithelium throughout the time lapse experiment. Counting was performed manually using ImageJ. Lung buds were categorized as peripheral branches only if they appeared at the most distal end of the secondary bronchi. Epithelial area was measured using the Keyence software. The epithelial area of lungs was marked by setting a threshold value to separate the pixels of the lighter epithelial tissue from the darker mesenchyme tissue, with corrections by hand as necessary. Once a threshold was determined, the total area of the epithelium and the perimeter was then measured and recorded. Statistical significance was determined using a two-tailed t-test with significance at $p<0.05$.

RNA isolation and double stranded cDNA synthesis. Total RNA was isolated from whole embryonic lungs at E18.5 using the RNeasy Mini Kit (Qiagen, Valencia, CA, USA), according to the manufacturer's instructions. Double stranded cDNA was synthesized from total mice lungs RNA using the iScriptTM cDNA Synthesis Kit from BIORAD following the manufacturer's instructions.

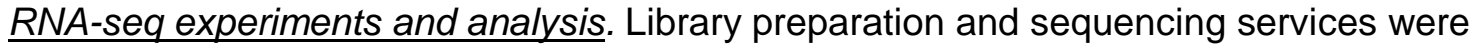
provided by Novogene Co. Ltd on the Illumina sequencing platform. RNA samples were processed by Novogene according to manufacturer instructions. The generated cDNA library was sequenced in $2 \square \times \square 150 \square$ bp paired-end layout using Illumina HiSeq2500. Sequencing data were analyzed using the Galaxy web platform (https://usegalaxy.org/) ${ }^{50}$. Sequence quality was assessed via FastQC (version 0.11.8) ${ }^{51}$. Sequence reads were aligned to the reference mouse genome (GRCm38) using STAR (default parameters, version 2.6.0b-1) ${ }^{52}$. Mapped reads were tallied using featureCounts Galaxy Version 1.6.4 (default parameters). Differential gene expression analysis was performed using DESeq2 (version 1.22.1) ${ }^{53}$ in the R programming environment (version 3.5.1) ${ }^{54}$. Pathway and function enrichment analysis on the differentially expressed genes was performed using metascape (http://metascape.org) ${ }^{55}$. Results were visualized as an enrichment network and enrichment bar graph. Annotation enrichment analysis on the differentially expressed genes was performed with VisuaL Annotation Display (VLAD) ${ }^{56}$ and with the Gene Ontology term enrichment tool (http://geneontology.org/docs/go-enrichmentanalysis/).

Quantitative PCR. After obtaining cDNA, real-time PCR was performed with IQ SYBR Green Supermix (Biorad,) and gene-specific primers. The data were analyzed using the $\Delta \Delta \mathrm{Ct}$ method on the BioRad CFX manager v1.5. RT-qPCR mRNA expression was analyzed by the Wilcoxon rank-sum under the hypothesis of $\mathrm{H} 1=\mathrm{a}<\mathrm{b}$. We reasoned $\mathrm{H} 1$ as RT-qPCR assay followed protein cell counting in which we already predicted that $a<b$.

The following primer pairs were used for qPCR of mouse genes with Actb as the reference for normalization: 
Sox9: 5' AGGAAGTCGGTGAAGAACGG3', 5'GGACCCTGAGATTGCCCAGA3' Fgfr2: 5' TGTGCAGATGGGATTACCGT3', 5' ATTTGGTTGGTGGCTCTTCTG3' Kras: 5'GATGTGCCTATGGTCCTGGTA3', 5'GCATCGTCAACACCCTGTCT3' Fgfr2b: 5'AGCTCCAATGCAGAAGTGC TGG 3', 5' GTTTGGGCAGGACAGTGAGCC 3'; Fgfr2c: 5' CCACGGACAAAGAGATTGAGG 3', 5' TGTCAACCATGCA GAGTGAAAG 3'; Actb: 5' CGGCCAGGTCATCACTATTGGCAAC3', 5' GCCACAGGATTCCATACCCAAGAAG3'.

scRNA-Seq single cell suspensions. To prepare the single cell suspensions, a pregnant, heterozygous female Svep1 mouse (JAX 023814) was sacrificed at 18.5 days post coitum (dpc) by cervical dislocation (IACUC protocol 10100 to CJB). Embryos were removed from the uterine horn and transferred to dishes of warm $\left(37^{\circ} \mathrm{C}\right) 1 \mathrm{X}$ Delbecco's Phosphate Buffered Saline (DPBS; ThermoFisher, 14190250 ) and euthanized by decapitation. Tail tips from each embryo were collected for genotyping analysis. The distal tip of the right caudal lung lobe from each embryo was resected and transferred to individual wells of a 12 well plate. The methods for single-cell suspension preparation were adapted from Sekiguchi and Hauser ${ }^{57}$. Each tip was submerged in a cold solution of $40 \mathrm{uL}$ of $1.6 \mathrm{U} / \mathrm{mL}$ dispase II (ThermoFisher, 17105041) and 40 $\mu \mathrm{L}$ Dulbecco's Modified Eagle Medium (DMEM)/F12 (ThermoFisher, 11039021) and incubated for 10 minutes at $37^{\circ} \mathrm{C}$ and $5 \% \mathrm{CO} 2$. After incubation, dispase was inactivated by adding $80 \mu \mathrm{L}$ of cold DMEM/12 supplemented with $5 \%$ bovine serum albumin (BSA; Sigma-Aldrich, A8577) into each well. The lung tissues were then transferred into individual wells of a new 12 well dish, each well of which contained $80 \mu \mathrm{L}$ of calcium and magnesium free Hanks' Balanced Salt Solution (HBSS, ThermoFisher, 14170112) to rinse off the media. Individual tissue pieces were then transferred to separate $1.5 \mathrm{~mL}$ microcentrifuge tubes (USA Scientific, 1415-2500), each containing $80 \mu \mathrm{L}$ protease mix $(4.5 \mu \mathrm{L} / \mathrm{mL}$ Accutase; Stem Cell Technologies, $07920 ; 4.5 \mu \mathrm{L} / \mathrm{mL}$ Accumax; Stem Cell Technologies, 07921, and 0.1mg/mL Bacillus licheniformis protease; Creative Enzymes, NATE0633, in calcium/magnesium-free DPBS). Samples were gently agitated with a pipet to dissociate for 2 minutes. Samples were then incubated on ice for 15 minutes before addition of $920 \mu \mathrm{L}$ calcium and magnesium free DPBS supplemented with $10 \%$ filtered Fetal Bovine Serum (FBS, Millipore Sigma)The samples were then filtered using Flowmi 40-um strainers (Bel-Art, H13680-0040) into individual Corning 15-mL tubes. 500 $\mu \mathrm{L}$ of DPBS plus $10 \%$ FBS was added to each sample and samples were centrifuged at $112 \mathrm{xg}$ for 7 minutes at room temperature. Supernatants were carefully removed and cell pellets were each washed with $1 \mathrm{~mL}$ of calcium and magnesium-free DPBS plus $1 \%$ FBS. Samples were centrifuged at $112 x g$ for 4 minutes at room temperature then all but $40-50 \mu \mathrm{L}$ of each supernatant was removed.

Following filtration, single cell suspensions from two mutant and two wild type Svep1 embryos were immediately washed and resuspended in PBS containing $0.04 \%$ BSA and cells were counted in a Countess II automated cell counter (ThermoFisher). From each sample, 12,000 cells were loaded into one lane of a 10X Chromium microfluidic chip. Single cell capture, barcoding, and library preparation were performed using the 10x Chromium version 3 chemistry kit according to the manufacturer's protocol (\#CG00183). Quality checks for cDNA and libraries were conducted on an Agilent 4200 TapeStation. The cDNA and libraries were then quantified 
using KAPA Biosystems qPCR (Sigma-Aldrich) and sequenced using Novaseq6000 (Illumina) to an average depth of 50,000 reads per cell.

scRNA-seq library preparation and quality control. Raw sequencing files were demultiplexed and FASTQ files were generated using the Cell Ranger analysis pipeline from 10x genomics (https://support.10xgenomics.com/single-cell-gene-expression/software/pipelines/latest/what-iscell-ranger). Sequencing reads with mismatches within the eight-base i7 Index 1 were filtered out. The remaining libraries were aligned to the mouse reference genome assembly (GRCm38.p6) using STAR ${ }^{52}$. Sequence reads with MAPQ scores less than 255 or containing bases with Q30 scores below 3 were removed. Following alignment, the cell bar codes were mapped to a list of 737,500 barcodes from 10X Genomics, with one allowed mismatch. Cell barcodes associated with a unique molecule identifier (UMI) count lower than the threshold were assumed to be related to ambient mRNA and were filtered out. A count matrix of the remaining cells and their associated genes was generated by CellRanger (10X Genomics) and used for downstream analysis. A total of 6,864 cells from mutant samples and 12,309 cells from the wild type Svep1 controls were characterized with 31,053 raw genes.

The Seurat R package was used to analyze the data ${ }^{58 ; 5960 ; 61}$. Each replicate and experimental group was analyzed prior to aggregation to check the quality of cells and cell populations. Within each of the replicates, cells were filtered based on mitochondrial RNA content, UMI counts, and high/low gene counts. To normalize RNA counts in each cell, RNA that mapped to the mouse genome was divided by total RNA counts for each cell, then scaled by a factor of 10000 and log transformed. After feature RNA normalization, replicates were aggregated into the two experimental groups.

scRNA-seq data processing. The two experimental groups were aggregated separately and scaled to account for batch effects between replicates. Linear regression was used to scale all 31,053 genes to preserve expression variance of low abundance genes from small populations of progenitor cells. Principal Component Analysis (PCA) was conducted to establish 80 principal components. Random resampling using Seurat's jackstraw function was used to calculate the significance of each PC ${ }^{62 ; 63}$. Clustering of cells was determined using the Jaccard index between each cell and its k-nearest neighbors. For the final dimension reduction step, Uniform Manifold Approximation and Projection (UMAP) was applied to the samples to project the data onto low-dimensional and easily visualized space while conserving global relationships ${ }^{64}$. Marker genes for each cluster were determined by running differential expression analysis using the Wilcoxon Rank Sum test on each cluster. To determine the cell types of each cluster, iterations of the $15,20,25$, and 30 most upregulated genes from each cluster were mapped to a lung cell population markers dataset curated from CellMarker and LungGENS ${ }^{65 ; 66}$. Clusters were labeled with the cell type according to the most upregulated genes associated with the cell type markers. The cell populations of each experimental group were visualized using UMAP.

The two normalized data sets were then merged using nearest neighbor cell pairs between the two experimental groups, or anchors, to ensure that similar cells from each group 
were mapped closely ${ }^{61 ; 67}$. Scaling, PCA, clustering, and UMAP dimension reduction analysis for the integrated data set were conducted in the same manner as the individual sample sets.

Segregation of mutant cells in various populations. To determine the cell populations most impacted by the loss of Svep1, the distribution of cell populations in each experimental group was determined by calculating the ratio of each Seurat cluster to total number of cells from an experimental group. A t-test of the ratios was conducted to determine populations significantly enriched or depleted in the mutant tissue. Clusters demonstrating a segregation between mutant and wild type origin cells were compared to the nearest-related clusters using the Wilcoxon Rank Sum test for differential gene expression.

Differential gene expression and annotation enrichment for scRNA-Seq. Differential gene expression was defined as genes with a p-value $<0.05$ expressed in at least $25 \%$ of the analyzed populations, and $-0.25>$ log fold change $>0.25$. Differentially expressed gene sets were submitted to the Visual Annotation Display (VLAD) ${ }^{56}$ software to perform annotation enrichment analysis for Mammalian Phenotype and Gene Ontology annotations.

\section{Results}

Svep1 is expressed in the lung mesenchyme with high expression at the lung periphery. To determine when and where Svep 1 is expressed during lung development, we performed immunohistochemistry and in situ hybridization of Svep1 at E12.5, E14.5, E16.5 and E18.5. During the pseudoglandular stage (E12.5-E16.5), Svep1 mRNA and protein expression were observed in the mesenchyme, especially at the tip and edges of the lung (Fig. 1A-C). Svep1 gene expression was strong adjacent to the distal epithelial airways and around bifurcating tips (Fig. 1A). SVEP1 protein labeling was observed at the distal airway epithelia buds, at bifurcating points, and in the developing microvasculature (Fig. 1C). In E12.5 (data not shown) and E14.5 lungs, we observed some SVEP1 protein adjacent to the epithelial membrane of very distal buds, co-localized with ECAD (Fig. 1B). At the saccular stage (E18.5), Svep1 mRNA and protein were localized in the primary septa of lung parenchyma and adjacent to the proximal epithelium (Fig. 1D). Consistent with previous findings, Svep1 expression during lung development was highest between E14.5 and E18.5 ${ }^{68}$.

Lungs of Svep1 knockout mice are hypoplastic and have abnormal lobes, a hypercellular mesothelium, and small saccules. Consistent with previous reports, Svep ${ }^{-/}$embryos displayed perinatal lethality, whole-body edema, and lung hypoplasia that was prominent at E18.5 by microCT (Fig. 1E) ${ }^{44}$. The absence of Svep1 expression in the lungs of homozygous knockout animals was confirmed by in situ hybridization (data not shown). The ventral side of the lungs of Svep 1/- embryos at E18.5 had small and rounded lobes that failed to surround the heart and the lung surface in that region was heavily lobulated giving the lung lobes a cauliflower-like appearance (Fig. 1F). Histological analysis of the lungs of mutant mice showed that the lung surface had a serrated appearance by E14.5 and lungs were obviously hypoplastic by E16.5 (Fig. 1G). At E18.5, the lungs of Svep1 mutants exhibited small saccules along a dorsal-ventral 
gradient, with a reduced lumen primarily on the lobulated ventral side of the lung $(\mathrm{Fig} .1 \mathrm{H})$. Lungs of Svep $1^{-/}$embryos exhibited a hypercellular mesothelium (Fig. 1H).

Saccular development begins in the murine lung shortly after E16.5, with the flattening of pneumocytes and the proximal-to-distal opening of the distal airway lumen. At the lung edge, distal airways are the last to differentiate. These distal airways retain a progenitor state and remain closed $^{28 ; 69}$. To examine airway morphology, we labeled the lung epithelium with both ECAD (Cdh1; MGl:88354) and the distal progenitor marker, SRY-box 9 (Sox9; MGl:98371). The images showed multiple, densely packed distal airways in the distal tip in E16.5 mutant lungs (Fig. 1G). Labeling at E18.5 demonstrated that the distal airway epithelium in the lobulated Svep $1^{-/}$lungs retained a typical pseudoglandular stage epithelial cuboidal form and expressed high levels of SOX9 (Fig. 1H). KI67 staining confirmed that the SOX9+ cells were actively proliferating (data not shown). Quantitative real-time PCR (qPCR) also confirmed a significant increase of Sox9 expression in the lungs of Svep1 knockout embryos at E18.5 (data not shown).

\section{Lungs of Svep 1/- embryos are characterized by an aberrant increase and disorganization of} distal airways which affects the shape of lung lobes. We used immunofluorescence imaging with SOX9 to evaluate the distal airways in individual lung lobes of Svep1 wild type and mutant embryos from E13.5 and E14.5 (Fig. 2A-D). We found branching and lobe shape anomalies in Svep $1^{-/}$lungs as early as E13.5, primarily at the lung tips and edges, which became more severe as lung development progressed. The lobe shape anomalies were more pronounced in the Right caudal (RCa) lobes compared to the other lobes in Svep $1^{-1 /}$ embryos (Fig.2A). The Right Accessory (RAc) lobe, which normally ends in a single tip around E13.5, often terminated in two or more airways in the lungs of mutant embryos. The accessory lobes in mutant were shorter than in wild type embryos (Fig. 2A). We also observed secondary ectopic lateral budding in the RAc lobe of the mutants (Fig. 2B).

During normal planar bifurcation single distal tips flatten, form a cleft, and then bifurcate into two daughter branches ${ }^{4}$. According to our findings at E13.5, Svep1 deficiency caused branching anomalies in the lung edges and tips, including mis-localized and extra budding (Fig. $2 A, B)$. In contrast to typical pointed lobes in wild type mice, the left lobes in mutant embryos are wider due to aberrant distal tip patterns which appear to be caused by defects in the branching angles (Fig. 2C).

The orthogonal bifurcation mode of the branching program fills the lung's interior in a specific pattern and creates the lobe surface of the lung lobes ${ }^{4}$. In lungs of mutants at E14.5, the airways were rearranged in a "row of beads" pattern leading to abnormal rounded tips (Fig. 2C). In addition, the left lung lobes of mutant embryos were $8 \%$ longer than wild type Svep1 embryos ( $p<0.001$ t-test). Whole left lobes cleared and labeled with SOX9 at E14.5 confirmed abnormal mutant left lobe morphology due to distal airway multi-budding and peripheral trifurcations (Fig. 2D). Defects in branching were also reflected in the significant increase in the number of distal tips SOX9+ at E14.5 (Fig. 2E; $p<0.05$ t-test). Taken all together, these results 
suggest that Svep1 plays a critical role the branching program and determines the architecture of the bronchial tree and consequently, the shape of lung lobes.

Lungs of Svep $1^{-}$embryos have ectopic branching, decreased bifurcations, and increased trifurcations. To determine which of the main branching programs are distorted in Svep1 mutants, we modeled the branching patterns of normal and mutant lungs. To study the structure of the airway epithelium at E14.5, we labeled tissue-cleared whole lungs with ECAD and SOX9 and generated a 3D reconstruction composed of Z-stack images. Our findings revealed significant structural differences in the airways of wild type and Svep $1^{-/}$lungs, confirming the duplication of the distal airway at the lobe tip as well as the disorder of the whole airway tree (Fig. 3).

To quantify differences in branching morphology in mutant vs wild type embryos we generated a branching lineage diagram for the right accessory (RAc) lobe at E14.5. Each branch was characterized as one of three branching modes: domain, planar, or orthogonal ${ }^{4}$. Branches and direction of growth were mapped to create a lineage diagram with trifurcations indicated (Fig. 3B). Orthogonal branching was significantly increased in mutants, contributing $63 \%$ of all branches compared to the $49.19 \%$ ( $p<0.01, t$ test) in the lungs of Svep $1^{+/+}$embryos. In contrast, the planar branching in the RAc was significantly decreased in mutant $(5 \%)$ compared to normal (13\%) embryos ( $p<0.05, t$ test). An average of $17 \%$ of first-level domain branches originating from the primary bronchus were ectopic in Svep $1^{-1}$ lungs, while no ectopic branching was observed in the lungs of wild type embryos (Fig. 3D). Trifurcations were more prevalent in Svep $1^{-/}$lungs (10\% of all branches) compared to the lungs of Svep $1^{+/}$embryos ( $4 \%$ of all branches) ( $p<0.05$ t test; Fig. $3 C$ ). In RAc lobes of normal lungs, daughter branches of the primary bronchus sprouted from one of only three directions: anterior, ventral-posterior, and dorsal-posterior ${ }^{4}$. In all the Svep $1^{-/}$lungs analyzed, daughter branches sprouted from the primary bronchus in random directions (Fig. 3D).

To determine if the ectopic and trifurcated branches in Svep $1^{-1}$ lungs was the result of loss of Svep1, we analyzed time-lapse images of E12.5 lung explants for $48 \mathrm{hr}$ (Fig. 3E ). We observed ectopic branching arising from the main and secondary branches and numerous trifurcations at the lung tips. From this data we conclude that Svep1 plays a role in determining the location and branching mode of new airway epithelial buds, which affects the shape and structure of the airway epithelium in the developing lung.

Single Cell RNA sequencing (scRNA-seq) of the distal lung identifies cell populations depleted in mutant mice. To investigate saccule development and its effect on cell populations and pathways in lung development, we conducted single cell RNA sequencing (scRNA-seq) and bulk RNA sequencing (RNA-seq) on the distal tip of right caudal lobe from wild type and mutant embryos at E18.5. We isolated 6,864 cells from mutant samples and 12,309 cells from the wild type Svep1 controls, characterized with 31,053 genes. After performing quality control and merging the individual single cell sequencing data sets, we conducted scaling of the total data set using a linear transformation to preserve the expression patterns of genes with low levels of global expression. UMAP analysis of the top 80 Principal Components revealed 24 cell clusters 
representing 11 cell types: matrix fibroblast cells, type I pneumocytes, type II pneumocytes, smooth muscle cells, ciliated cells, pericytes, myeloid cells, fibroblasts, epithelial cells, endothelial cells, and club cells (data not shown). Svep1 is expressed predominantly in matrix fibroblast cell populations. Analysis of functional annotation terms for differentially expressed genes between the lungs of Svep $1^{+/+}$and Svep $1^{-/}$embryos from both bulk and scRNA Seq data revealed an enrichment of biological process and phenotype terms associated with cell mobility and adhesion, vasculature development, and extracellular matrix formation (data not shown). These findings are consistent with previous studies on phenotypes observed in mice as a consequence of the loss of Svep 1 function in a variety of tissues and cell types ${ }^{39-41 ; 70}$.

Svep1 is necessary for alveolar epithelial cellular differentiation and maturation. The small or absent saccules in mutants and the increase of SOX9+ cells (Fig. 1H), suggest that a persistent branching program in Svep1 mutants is blocking normal alveolar differentiation. Lung sections stained with toluidine blue at E18.5 revealed a high density of round type II pneumocytes, small saccules, and a reduced expression of surfactant proteins in the lungs of Svep 1/ embryos (Fig. 5A). Notably, scRNA Seq data revealed that type I pneumocytes (AT1) cells represented 5.1\% of the total cell composition in the lungs of $S v e p 1^{+/+}$embryos, but only $2 \%$ of the total population for the lungs of Svep $1^{-/}$embryos (data not shown). Genes associated with type 1 pneumocytes (AT1) were found to be significantly lower in the lungs of Svep $1^{-1-}$ mutant embryos (4B) whereas genes associated with type II pneumocytes (AT1) were upregulated (Fig. 4B). To evaluate type I pneumocyte development in the developing lung, we labeled E18.5 lungs with type 1 pneumocyte markers PDPL and HOPX (4C,D). In Svep 1\% embryos, PDPL and HOPX1 positive domains were reduced by E16.5 (data not shown) and in the small alveolar saccules at E18.5 (Fig. 4C,D). These findings suggest that in the absence of Svep1, the alveolar epithelium cannot differentiate and mature correctly.

The loss of Svep1 leads to defects in smooth muscle in the lung. Several muscle development related genes were significantly downregulated in the lungs of Svep $1^{-/-}$embryos (Fig. 5A), including Acta2 (actin 2; MGl:87909) which is necessary for smooth muscle differentiation. Cnn1 (calponin1; MGl:104979), a marker for mature smooth muscle, was also downregulated in mutants (Fig. 5A). Lungs labeled with ACTA2 at E18.5 confirmed the reduction in distal bronchioles in the mutant lung at E16.5 (Fig. 5B). Blood vessels adjacent to the conductive airways also showed a reduction in ACTA2 labeling in the lungs of Svep $1^{-/}$embryos (Fig. 5B). Defects in smooth muscle differentiation and airway formation were confirmed by ex vivo organ culture of E12.5 Svep 1\% when lung explants were allowed to grow in vitro for six days (Fig. 5C). The expression of ACTA2 in the explant cultures was noticeably reduced in mutants, particularly in lobular bronchioles.

Alveolar myofibroblasts are ACTA2 positive cells that transiently differentiate from their PDGFRA (MGI:97530) positive progenitors during septation ${ }^{71}$. During normal murine lung development, PDGFRA progenitors are located at the base of saccules in the regions of future alveoli and migrate toward the saccule lumen ${ }^{72}$. Immunofluorescence of PDGFRA and ACTA2 positive cells revealed a deficiency in alveolar myofibroblast (ACTA2+), but not in their progenitors (PDGFRA+), in the lungs of E18.5 Svep1/- embryos (Fig. 5D). Quantification of 
PDGFRA+ cells revealed that the average number of PDGFRA+ cells was higher in lungs of Svep $1^{-/}$embryos at E18.5 compared to wild type $\left(\right.$Svep $1^{+/+}=115.8 \pm 7.1$; Svep $1^{-/}=178.3 \pm 23.0$; $\mathrm{p}<0.05$ t-test). Also, the ratio of ACTA2+ to PDGRFA+ cells was lower (Svep1 $1^{+/+}=49.2 \pm 2.2$; Svep1-/- = $29.2 \pm 4.8 ; p<0.05$ t-test) in mutants (Fig. 5E). No significant differences in the expression of Pdgfra in mutant and wild type lungs were detected in the RNA Seq data. However, Pdgfa (MGI:97527) expression was reduced in Svep1\% embryos at E18.5 (Log2(FC)= - 0.19318, adjusted $p$ value <0.05). PDGFA signals through PDGFRA and is involved in myofibroblast proliferation and migration ${ }^{73 ; 74}$. Pdgfra+ cells give rise also to alveolar lipofibroblasts that are characterized by their lipid-filled vesicles ${ }^{75 ; 76}$. We looked at the expression of Plin2 (perilipin 1 -- aka adipophilin; MGI:87920) in the RNA Seq data to determine if alveolar fibroblast development was biased toward lipofibroblast development but there were no significant differences in expression for this gene between mutants and wild type lungs at E18.5. These data are consistent with the hypothesis that Svep1 is required for differentiation of smooth muscle cells, particularly in the distal lung, beginning early in lung development.

Conductive airways in Svep $1^{-/}$embryos are hypoplastic and enriched in basal cells. Club cells are secretory cells abundant in terminal bronchioles as a source of progenitors capable of differentiation into ciliated and goblet cells during normal lung development ${ }^{11}$. The scRNA Seq data indicated that the cluster of Club cells was enriched in cells from Svep1 mutants relative to wild type (data not shown). To determine mutant defects in epithelial airways differentiation, we labeled the proximal epithelium with the club cell marker, Secretoglobin 1a1 (Scgb1a1; MGI:98919), and a marker of ciliated cells, Forkhead box protein J1 (Foxj1; MGI:1347474) (Fig. 6A). Svep 1\% embryos labeled with SCGB1A1 displayed a hypoplastic distal airway with a narrow lumen compared to controls (Fig. 6A). The bronchioles in mutant embryos were hypercellular and often displayed a disorganized epithelium (Fig. 6A). At E18.5, most epithelial cells in distal bronchioles of normal murine lungs are non-ciliated cuboidal cells positive for SCGB1A $1^{77}$. We quantified the total number of SCGB1A1+ and FOXJ1+ cells and calculated the ratio of these cells in the terminal bronchioles of normal and mutant lungs. The number of SCGBA1+ cells was significantly increased in mutants, but the total number of FOXJ1+ cells and the ratio between SCGBA1+ and FOXJ1+ were not statistically significant (Fig. 6B). The expression of Scgba1 in mutants did not differ from that of wild-type mice (data not shown).

Svep $1^{-1}$ lungs exhibit disorganized vasculature and mesothelial immaturity. Both the scRNA Seq and bulk RNA Seq data revealed that the lungs of Svep1 knockout embryos have decreased expression of vascular development regulators including annexin A1 (Anxa1; MGI:96819) and chitinase like-1 (Chil1; MGl:12654). These results are consistent with previous work showing the role of Svep1 in vascular development and remodeling in the mouse ${ }^{40 ; 41}$. In addition, the cluster of pericyte cell type from the scRNA Seq data were depleted of mutant cells (data not shown). Annotation enrichment analysis also indicated that pathways associated with vascular function were disrupted in the mutants (data not shown). To determine if the lung microvasculature formation is affected in Svep $1^{-/}$lungs, we labeled E18.5 endothelial cells with the nuclear marker ETS-related gene (Erg; MGI:95415) (Fig. 6C). The microvasculature in the lungs of mutant embryos was disorganized and failed to form the double layer pattern characteristics of the saccular stage (Fig. 6C). 
The lungs are covered by a monolayer of progenitor mesothelial cells that are round and smooth and flatten when differentiated ${ }^{78}$. We observed that lungs from Svep ${ }^{-1}$ embryos at E18.5 had hypercellular edges and labeled lungs with the mesothelial progenitor marker Wilms' tumor suppressor 1 (Wt1; MGI:98968) (Fig 6D). Labeling with WT1 showed that the rounded lung lobes observed in mutant embryos were covered with mesothelial progenitors. The RNA Seq data showed that expression of Wt1 was higher in the lungs of the mutants (Fig. 6D,E). To determine if the increase of mesothelial cells was due to an increase in cellular proliferation, we co-labeled with WT1 and KI67 (aka Mki67; MGl:106035) (Fig. 6F). There was a significant increase in the number of WT1+ cells in Svep $1^{-/}$lungs $\left(\right.$Svep $1^{+/+}=43.0 \pm 7.0$; Svep $1^{-1-}=72.8 \pm$ 39.6; $\mathrm{p}<0.05 \mathrm{t}$ test). However, the percentage of $\mathrm{WT} 1^{+}$that were also $\mathrm{KI} 7^{+}$was not significantly different between normal and mutants at E18.5 $\left(\right.$ Svep $1^{+/+}=14.9 \pm 0.4$; Svep $\left.1^{-/}=13.1 \pm 0.6\right)$ (Fig. $6 \mathrm{E})$. This ratio was also not statistically different at E16.5 $\left(\right.$ Svep $1^{+/+}=25.2 \pm 0.9$; Svep $1^{-/-}=27.5 \pm$ 4.4). These results suggests that mesothelial differentiation is disrupted in mutants and that the observed phenotype is not due to increased proliferation.

SVEP1 acts as a negative regulator of branching morphogenesis. The increased branching observed in the distal airways of Svep $1^{-/}$embryos suggests that SVEP1 normally acts as a negative regulator of lung branching morphogenesis and is essential for regulating lung airway budding. To investigate the function of Svep1 in branching morphogenesis further, we treated lung explants from Svep $1^{-/}$and wild type E12.5 embryos for 48hr with a recombinant SVEP1 (rSVEP1) protein. Time lapse images revealed decreased branching in the treated lung explants (Fig. 7A). Quantification of peripheral branches revealed a significant decrease in branching at the tips of the lungs (Fig. 7B). Additional morphometric analysis revealed a much reduced epithelial area, a significantly decreased epithelial width, and an increase in bud elongation in E12.5 lung explants from Svep1 mutants (data not shown). When lung explants from E12.5 Svep $1^{-/}$embryos were treated with rSVEP we observed a dramatic reduction of ectopic and trifurcated branches; distal trifurcations were observed in only one of the six explants treated with rSVEP1 (Fig. 7C).

Branching patterning defects, but not smooth muscle differentiation, in Svep $1^{-/}$embryos are independent of the FGF pathway. Given the abnormal branching and the bulk RNA Seq data which revealed a significant upregulation in the expression of Fgfr2 in Svep 1/- lungs at E18.5 $($ Log2(FC) $=-0.722 ; \mathrm{P}$. value adj=6.66E-13), we investigated whether the FGF pathway is involved in the abnormal lung branching phenotype in Svep ${ }^{-/}$mutants. Fgfr2 is normally expressed in the lung epithelium with a decrease in expression after E17.5 ${ }^{68}$ In Svep1 ${ }^{-1}$ embryos however, in situ hybridization showed that levels of expression of Fgfr2 persisted in the lungs of mutants even at E18.5 compared to levels in Svep $1^{+/+}$lungs (Fig. 7D).

Although the expression of Fgf9 and Fgf10 was not significantly increased in E18.5 lungs of mutants based on bulk RNA Seq or scRNA Seq experiments (data not shown), RT-qPCR results showed a significant increase of Fgfr2 (MGl:95523), Fgf10 (MGl:1099809), Fgf9 (MGl:104723), and Kras (MGl:96680) in the lungs of Svep1 mutants E18.5 (Fig. 7E) but not at E14.5 or E16.5 (data not shown). Fgfr2 has two isoforms, Fgfr2b and Fgfr2c. Only the transcript abundance of Fgfr2b was significant at E18.5 (Fig. 7E). 
The airway smooth muscle in the lung develops from local mesenchymal cells located around the tips of growing epithelial buds. These cells become gradually displaced from distal to a proximal position alongside the bronchial tree, where they elongate, and begin to synthesize smooth muscle-specific proteins ${ }^{79}$. FGF10+ cells serve as progenitors to parabronchial smooth muscle cells and this progenitor status is maintained by mesothelial-derived FGF9 which has been shown to inhibit smooth muscle differentiation in the mouse lung ${ }^{80 ; 81}$. To investigate the involvement of the FGF pathway in Svep $1^{-/}$branching defects and smooth muscle differentiation further, we treated lung explants with the FGF pathway inhibitor SU5402 at a dose low enough to allow some branching activity. As expected, we observed some branching inhibition in the control and Svep $1^{-/-}$lung explants after $48 \mathrm{hr}$ of treatment (Fig. 7F). However, immunofluorescence of ACTA2 and SOX9 revealed distal trifurcations and first branch ectopic branching in Svep 1/- lungs (Fig. 7F). Lung explants treated with SU5402 for four days showed expanded ACTA2 localization encapsulating the distal airways of control and Svep ${ }^{-/}$lungs, as previously observed ${ }^{81 ; 82}$, but less so in mutants (Fig. 7G). Overall, these results indicate that the branching pattern defects in Svep1 mutants are independent of the FGF pathway, but that smooth muscle defects are, in part, mediated by increased FGF signaling in Svep1 mutants.

\section{Discussion}

Many previous studies of mammalian lung development have generated insights into the genetic and molecular processes that underlie lung branching morphogenesis and alveolarization. However, the factors that regulate the bronchial tree's stereotyped structure and the subsequent transition from branching to saccular formation are still unclear. This transition is essential for establishing the developmental foundation for alveologenesis and has significant clinical ramifications for neonates born with such respiratory deficiencies as lung hypoplasia. The study reported here demonstrated, for the first time, that the extracellular matrix gene, Svep1, is necessary for ending lung branching morphogenesis and that the loss of Svep1 impairs alveolar maturation and differentiation.

Key findings of this study are that Svep1 is essential for normal lung branching and acts as a negative regulator of branching. In Svep 1/ embryos on a C57BL/6J inbred background, abnormalities of the lung structure were obvious by E13.5 and became progressively worse over the course of development. Svep1 is normally highly expressed in the mesenchyme of bifurcating peripheral lung tips during branching and these regions were most impacted by the loss of Svep1. The absence of Svep1 led to marked lung hypoplasia but with a concomitant increase in branching in the distal airways. Normally, lung buds tend to fill the mesenchyme homogeneously in an orderly and non-conflicting or overlapping pattern ${ }^{5}$. The lungs of Svep $1^{-/}$ embryos, however, were characterized by ectopic branching, decreased bifurcations, and increased trifurcations which resulted in an increased number of disorganized distal airway tips and a disruption in the shape of the lung lobes. A 3D reconstruction of the right accessory (RAc) lobe revealed that the abnormal trifurcations in the branching results in a pattern of dense rosettes in the distal airways. This branching defect is most likely responsible for the cauliflowerlike appearance of Svep $1^{-/}$lungs. The impact of the loss of Svep1 was different for different 
lung lobes. Lobes with wide flat tips, such as the right caudal $(\mathrm{RCa})$ and right cranial $(\mathrm{RCr})$ lobes, were more elongated with rounded edges in the mutants compared to the lobes from wild type embryos which were flat at the edges and terminated pointed tip. The RAc lobe and right medial $(\mathrm{RMe})$ lobe were foreshortened in mutants with rounded tips, presumably a consequence of increased and disorganized distal branching.

As FGF signaling is known to be essential for branching morphogenesis ${ }^{83}$ we investigated the impact of inhibiting FGF signaling in lung explant cultures. Lungs from Svep $1^{-/}$embryos continued to exhibit distal airway trifurcation in absence of FGF, demonstrating that the action of Svep1 on branching is independent of FGF signaling. The abnormal branching phenotype in the lungs of Svep 1/ embryos was reversed by treating E12.5 lungs for 24 hours in organ culture with recombinant SVEP1 protein. Inhibition of SOX9, which is downstream of SVEP1, also resulted in marked reduction of abnormal branching (data not shown).

In addition to the lung branching defects in Svep1 mutants we observed defects in airway smooth muscle and microvasculature. Svep1 has been shown previously to be involved in the early steps of myoblast differentiation and epithermal differentiation ${ }^{39 ; 84}$. Our results suggests that Svep1 plays a role in the direct regulation of smooth muscle differentiation in the lung supported by the transcriptomics data that revealed a significant downregulation of a number of smooth muscle genes in the lungs of mutant embryos. The microvasculature in the lungs of mutant embryos was disorganized and failed to form the double layer pattern characteristics of the saccular stage. The results are consistent with prior work demonstrating the role of Svep1 in vascular system development and remodeling ${ }^{40 ; 41}$.

Another key finding of this study is that the lungs of Svep1 mutants exhibit impaired cellular differentiation. Embryos that lacked functional Svep1 died shortly after birth due to severe saccular defects. The lumens of the distal airways at E18.5 were reduced and had cell morphology more typical of the pseudoglandular stage. These cells also showed extensive positive labeling for SOX9+ progenitor cells. Differentiation of the distal epithelial was observed in the Svep1 mutant lungs but demonstrated a bias for type II (AT2) pneumocytes. These type II pneumocytes may have arisen from the proliferative SOX9${ }^{+}$cell population which, in turn, might be maintained by the abnormal persistent expression of genes involved in FGF signaling in Svep1 mutants. Svep 1/ lungs were deficient in type I (AT1) pneumocytes that arise from the $\mathrm{HOPX}^{+}$and $\mathrm{PDPL}^{+}$region near the stalk of the lung bud. Mesothelial cells at E18.5 in Svep $1^{-/}$ were also maintained in a progenitor state as evidenced by extensive positive labeling with the progenitor marker, WT1, and high expression of Wt1 in mutants compared to controls.

The results of the present study clearly demonstrate the importance of the extracellular matrix in branching patterning and alveolar cellular differentiation in the lung. Disruption of the extracellular matrix has been shown previously to be detrimental to cellular differentiation processes $^{85}$, but this is the first report of the role that Svep1 plays in orchestrating the transition from branching programs to alveolar differentiation. Additional investigation into the specific role that Svep1 plays during the saccular phase to prepare the lung for alveologenesis will be required to understand the detailed molecular and cellular mechanisms of this transition. Based 
on the observations in this study, we posit that SVEP1 peptides or inhibition of SVEP1 downstream targets such as SOX9 and members of the FGF signaling pathway could be useful approaches for treating lung prematurity. Dosage and timing of such interventions would need further study to determine regimens that reduce branching and simultaneously enhance differentiation and alveolarization. Studies of genes which, when disrupted, lead to congenital diaphragmatic hernia with a lung phenotype, could also be exploited to uncover therapeutics for treatment of respiratory anomalies characteristic of lung prematurity without CDH. Therapeutic modulation of the ECM may also open up new therapeutic avenues for treating lung damage arising from chronic obstructive pulmonary disease (COPD) or Covid19 infection where restoration of the regenerative capacity of the lung would be transformative in improving patient outcomes.

\section{Acknowledgements}

The authors thank Drs. Martin Ringwald and Vidhya Munnamalai for their comments on an early draft of this manuscript. Sandy Daigle (The Jackson Laboratory) provided essential technical support and advice for the scRNA Seq experiment. Pete Finger (The Jackson Laboratory) performed the toluidine blue staining.

\section{Funding}

Funding was provided by the National Institute of Child Health and Human Development (NICHD) P01HD068250.

\section{References}

1. Rawlins, E.L. (2011). The building blocks of mammalian lung development. Dev Dyn 240, 463-476.

2. Chang, D.R., Martinez Alanis, D., Miller, R.K., Ji, H., Akiyama, H., McCrea, P.D., and Chen, J. (2013). Lung epithelial branching program antagonizes alveolar differentiation. Proc Natl Acad Sci U S A 110, 18042-18051.

3. Warburton, D., El-Hashash, A., Carraro, G., Tiozzo, C., Sala, F., Rogers, O., De Langhe, S., Kemp, P.J., Riccardi, D., Torday, J., Bellusci, S., Shi, W., Lubkin, S.R., and Jesudason, E. (2010). Lung organogenesis. Curr Top Dev Biol 90, 73-158.

4. Metzger, R.J., Klein, O.D., Martin, G.R., and Krasnow, M.A. (2008). The branching programme of mouse lung development. Nature 453, 745-750.

5. Blanc, P., Coste, K., Pouchin, P., Azais, J.M., Blanchon, L., Gallot, D., and Sapin, V. (2012). A role for mesenchyme dynamics in mouse lung branching morphogenesis. PLoS One 7, e41643.

6. Zhao, X., Ju, Y., Liu, C., Li, J., Huang, M., Sun, J., and Wang, T. (2009). Bronchial anatomy of left lung: a study of multi-detector row CT. Surg Radiol Anat 31, 85-91.

7. Rawlins, E.L., Clark, C.P., Xue, Y., and Hogan, B.L. (2009). The Id2+ distal tip lung epithelium contains individual multipotent embryonic progenitor cells. Development 136, 3741-3745.

8. Frank, D.B., Penkala, I.J., Zepp, J.A., Sivakumar, A., Linares-Saldana, R., Zacharias, W.J., Stolz, K.G., Pankin, J., Lu, M., Wang, Q., Babu, A., Li, L., Zhou, S., Morley, M.P., Jain, R., and Morrisey, E.E. (2019). Early lineage specification defines alveolar epithelial ontogeny in the murine lung. Proc Natl Acad Sci U S A 116, 4362-4371. 
9. Yang, Y., Fu, Q., Liu, Y., Wang, X., Dunham, R., Liu, S., Bao, L., Zeng, Q., Zhou, T., Li, N., Qin, Z., Jiang, C., Gao, D., and Liu, Z. (2018). Comparative transcriptome analysis reveals conserved branching morphogenesis related genes involved in chamber formation of catfish swimbladder. Physiol Genomics 50, 67-76.

10. Conway, F.M., Garner, J.L., Orton, C.M., Srikanthan, K., Kemp, S.V., and Shah, P.L. (2019). Contemporary Concise Review 2018: Lung cancer and pleural disease. Respirology 24, 475-483.

11. Rock, J.R., and Hogan, B.L. (2011). Epithelial progenitor cells in lung development, maintenance, repair, and disease. Annu Rev Cell Dev Biol 27, 493-512.

12. Herriges, M., and Morrisey, E.E. (2014). Lung development: orchestrating the generation and regeneration of a complex organ. Development 141, 502-513.

13. Whitsett, J.A., Kalin, T.V., Xu, Y., and Kalinichenko, V.V. (2019). Building and Regenerating the Lung Cell by Cell. Physiol Rev 99, 513-554.

14. Morrisey, E.E., and Hogan, B.L. (2010). Preparing for the first breath: genetic and cellular mechanisms in lung development. Dev Cell 18, 8-23.

15. Bellusci, S., Grindley, J., Emoto, H., Itoh, N., and Hogan, B.L. (1997). Fibroblast growth factor 10 (FGF10) and branching morphogenesis in the embryonic mouse lung. Development 124, 4867-4878.

16. Min, H., Danilenko, D.M., Scully, S.A., Bolon, B., Ring, B.D., Tarpley, J.E., DeRose, M., and Simonet, W.S. (1998). Fgf-10 is required for both limb and lung development and exhibits striking functional similarity to Drosophila branchless. Genes Dev 12, 31563161.

17. Sekine, K., Ohuchi, H., Fujiwara, M., Yamasaki, M., Yoshizawa, T., Sato, T., Yagishita, N., Matsui, D., Koga, Y., Itoh, N., and Kato, S. (1999). Fgf10 is essential for limb and lung formation. Nat Genet 21, 138-141.

18. De Moerlooze, L., Spencer-Dene, B., Revest, J.M., Hajihosseini, M., Rosewell, I., and Dickson, C. (2000). An important role for the Illb isoform of fibroblast growth factor receptor 2 (FGFR2) in mesenchymal-epithelial signalling during mouse organogenesis. Development 127, 483-492.

19. Weaver, M., Dunn, N.R., and Hogan, B.L. (2000). Bmp4 and Fgf10 play opposing roles during lung bud morphogenesis. Development 127, 2695-2704.

20. Abler, L.L., Mansour, S.L., and Sun, X. (2009). Conditional gene inactivation reveals roles for Fgf10 and Fgfr2 in establishing a normal pattern of epithelial branching in the mouse lung. Dev Dyn 238, 1999-2013.

21. Ramasamy, S.K., Mailleux, A.A., Gupte, V.V., Mata, F., Sala, F.G., Veltmaat, J.M., Del Moral, P.M., De Langhe, S., Parsa, S., Kelly, L.K., Kelly, R., Shia, W., Keshet, E., Minoo, P., Warburton, D., and Bellusci, S. (2007). Fgf10 dosage is critical for the amplification of epithelial cell progenitors and for the formation of multiple mesenchymal lineages during lung development. Dev Biol 307, 237-247.

22. Warburton, D. (2008). Developmental biology: order in the lung. Nature 453, 733-735.

23. Hyatt, B.A., Shangguan, X., and Shannon, J.M. (2004). FGF-10 induces SP-C and Bmp4 and regulates proximal-distal patterning in embryonic tracheal epithelium. Am J Physiol Lung Cell Mol Physiol 287, L1116-1126.

24. Ornitz, D.M., and Yin, Y. (2012). Signaling networks regulating development of the lower respiratory tract. Cold Spring Harb Perspect Biol 4.

25. Volckaert, T., Campbell, A., Dill, E., Li, C., Minoo, P., and De Langhe, S. (2013). Localized Fgf10 expression is not required for lung branching morphogenesis but prevents differentiation of epithelial progenitors. Development 140, 3731-3742.

26. Park, W.Y., Miranda, B., Lebeche, D., Hashimoto, G., and Cardoso, W.V. (1998). FGF-10 is a chemotactic factor for distal epithelial buds during lung development. Dev Biol 201, 125-134. 
27. Warburton, D., Bellusci, S., De Langhe, S., Del Moral, P.M., Fleury, V., Mailleux, A., Tefft, D., Unbekandt, M., Wang, K., and Shi, W. (2005). Molecular mechanisms of early lung specification and branching morphogenesis. Pediatr Res 57, 26R-37R.

28. Li, J., Wang, Z., Chu, Q., Jiang, K., Li, J., and Tang, N. (2018). The Strength of Mechanical Forces Determines the Differentiation of Alveolar Epithelial Cells. Dev Cell 44, 297-312 e295.

29. Mailleux, A.A., Kelly, R., Veltmaat, J.M., De Langhe, S.P., Zaffran, S., Thiery, J.P., and Bellusci, S. (2005). Fgf10 expression identifies parabronchial smooth muscle cell progenitors and is required for their entry into the smooth muscle cell lineage. Development 132, 2157-2166.

30. Yuan, T., Volckaert, T., Chanda, D., Thannickal, V.J., and De Langhe, S.P. (2018). Fgf10 Signaling in Lung Development, Homeostasis, Disease, and Repair After Injury. Front Genet 9, 418.

31. Zhou, Y., Horowitz, J.C., Naba, A., Ambalavanan, N., Atabai, K., Balestrini, J., Bitterman, P.B., Corley, R.A., Ding, B.S., Engler, A.J., Hansen, K.C., Hagood, J.S., Kheradmand, F., Lin, Q.S., Neptune, E., Niklason, L., Ortiz, L.A., Parks, W.C., Tschumperlin, D.J., White, E.S., Chapman, H.A., and Thannickal, V.J. (2018). Extracellular matrix in lung development, homeostasis and disease. Matrix Biol 73, 77-104.

32. Mecham, R.P. (2018). Elastin in lung development and disease pathogenesis. Matrix Biol 73, 6-20.

33. Nguyen, N.M., Miner, J.H., Pierce, R.A., and Senior, R.M. (2002). Laminin alpha 5 is required for lobar septation and visceral pleural basement membrane formation in the developing mouse lung. Dev Biol 246, 231-244.

34. Sakai, T., Larsen, M., and Yamada, K.M. (2003). Fibronectin requirement in branching morphogenesis. Nature 423, 876-881.

35. Chen, J., and Krasnow, M.A. (2012). Integrin Beta 1 suppresses multilayering of a simple epithelium. PLoS One 7, e52886.

36. Loscertales, M., Nicolaou, F., Jeanne, M., Longoni, M., Gould, D.B., Sun, Y., Maalouf, F.I., Nagy, N., and Donahoe, P.K. (2016). Type IV collagen drives alveolar epithelialendothelial association and the morphogenetic movements of septation. BMC Biol 14, 59.

37. Walma, D.A.C., and Yamada, K.M. (2020). The extracellular matrix in development. Development 147.

38. Sato-Nishiuchi, R., Nakano, I., Ozawa, A., Sato, Y., Takeichi, M., Kiyozumi, D., Yamazaki, K., Yasunaga, T., Futaki, S., and Sekiguchi, K. (2012). Polydom/SVEP1 is a ligand for integrin alpha9beta1. J Biol Chem 287, 25615-25630.

39. Samuelov, L., Li, Q., Bochner, R., Najor, N.A., Albrecht, L., Malchin, N., Goldsmith, T., GrafiCohen, M., Vodo, D., Fainberg, G., Meilik, B., Goldberg, I., Warshauer, E., Rogers, T., Edie, S., Ishida-Yamamoto, A., Burzenski, L., Erez, N., Murray, S.A., Irvine, A.D., Shultz, L., Green, K.J., Uitto, J., Sprecher, E., and Sarig, O. (2017). SVEP1 plays a crucial role in epidermal differentiation. Exp Dermatol 26, 423-430.

40. Karpanen, T., Padberg, Y., van de Pavert, S.A., Dierkes, C., Morooka, N., Peterson-Maduro, J., van de Hoek, G., Adrian, M., Mochizuki, N., Sekiguchi, K., Kiefer, F., Schulte, D., and Schulte-Merker, S. (2017). An Evolutionarily Conserved Role for Polydom/Svep1 During Lymphatic Vessel Formation. Circ Res 120, 1263-1275.

41. Morooka, N., Futaki, S., Sato-Nishiuchi, R., Nishino, M., Totani, Y., Shimono, C., Nakano, I., Nakajima, H., Mochizuki, N., and Sekiguchi, K. (2017). Polydom Is an Extracellular Matrix Protein Involved in Lymphatic Vessel Remodeling. Circ Res 120, 1276-1288.

42. Blake, J.A., Baldarelli, R., Kadin, J.A., Richardson, J.E., Smith, C.L., Bult, C.J., and Mouse Genome Database, G. (2020). Mouse Genome Database (MGD): Knowledgebase for mouse-human comparative biology. Nucleic Acids Res. 
43. Kishore, R., Arnaboldi, V., Van Slyke, C.E., Chan, J., Nash, R.S., Urbano, J.M., Dolan, M.E., Engel, S.R., Shimoyama, M., Sternberg, P.W., and Genome Resources, T.A.O. (2020). Automated generation of gene summaries at the Alliance of Genome Resources. Database (Oxford) 2020.

44. Dickinson, M.E., Flenniken, A.M., Ji, X., Teboul, L., Wong, M.D., White, J.K., Meehan, T.F., Weninger, W.J., Westerberg, H., Adissu, H., Baker, C.N., Bower, L., Brown, J.M., Caddle, L.B., Chiani, F., Clary, D., Cleak, J., Daly, M.J., Denegre, J.M., Doe, B., Dolan, M.E., Edie, S.M., Fuchs, H., Gailus-Durner, V., Galli, A., Gambadoro, A., Gallegos, J., Guo, S., Horner, N.R., Hsu, C.W., Johnson, S.J., Kalaga, S., Keith, L.C., Lanoue, L., Lawson, T.N., Lek, M., Mark, M., Marschall, S., Mason, J., McElwee, M.L., Newbigging, S., Nutter, L.M., Peterson, K.A., Ramirez-Solis, R., Rowland, D.J., Ryder, E., Samocha, K.E., Seavitt, J.R., Selloum, M., Szoke-Kovacs, Z., Tamura, M., Trainor, A.G., Tudose, I., Wakana, S., Warren, J., Wendling, O., West, D.B., Wong, L., Yoshiki, A., International Mouse Phenotyping, C., Jackson, L., Infrastructure Nationale Phenomin, I.C.d.I.S., Charles River, L., Harwell, M.R.C., Toronto Centre for, P., Wellcome Trust Sanger, I., Center, R.B., MacArthur, D.G., Tocchini-Valentini, G.P., Gao, X., Flicek, P., Bradley, A., Skarnes, W.C., Justice, M.J., Parkinson, H.E., Moore, M., Wells, S., Braun, R.E., Svenson, K.L., de Angelis, M.H., Herault, Y., Mohun, T., Mallon, A.M., Henkelman, R.M., Brown, S.D., Adams, D.J., Lloyd, K.C., McKerlie, C., Beaudet, A.L., Bucan, M., and Murray, S.A. (2016). High-throughput discovery of novel developmental phenotypes. Nature 537, 508-514.

45. Skarnes, W.C., Rosen, B., West, A.P., Koutsourakis, M., Bushell, W., lyer, V., Mujica, A.O., Thomas, M., Harrow, J., Cox, T., Jackson, D., Severin, J., Biggs, P., Fu, J., Nefedov, M., de Jong, P.J., Stewart, A.F., and Bradley, A. (2011). A conditional knockout resource for the genome-wide study of mouse gene function. Nature 474, 337-342.

46. Truett, G.E., Heeger, P., Mynatt, R.L., Truett, A.A., Walker, J.A., and Warman, M.L. (2000). Preparation of PCR-quality mouse genomic DNA with hot sodium hydroxide and tris (HotSHOT). Biotechniques 29, 52, 54.

47. Ye, J., Coulouris, G., Zaretskaya, I., Cutcutache, I., Rozen, S., and Madden, T.L. (2012). Primer-BLAST: a tool to design target-specific primers for polymerase chain reaction. BMC Bioinformatics 13, 134.

48. Riddle, R.D., Johnson, R.L., Laufer, E., and Tabin, C. (1993). Sonic hedgehog mediates the polarizing activity of the ZPA. Cell 75, 1401-1416.

49. Schneider, C.A., Rasband, W.S., and Eliceiri, K.W. (2012). NIH Image to ImageJ: 25 years of image analysis. Nat Methods 9, 671-675.

50. Afgan, E., Baker, D., Batut, B., van den Beek, M., Bouvier, D., Cech, M., Chilton, J., Clements, D., Coraor, N., Gruning, B.A., Guerler, A., Hillman-Jackson, J., Hiltemann, S., Jalili, V., Rasche, H., Soranzo, N., Goecks, J., Taylor, J., Nekrutenko, A., and Blankenberg, D. (2018). The Galaxy platform for accessible, reproducible and collaborative biomedical analyses: 2018 update. Nucleic Acids Res 46, W537-W544.

51. Wingett, S.W., and Andrews, S. (2018). FastQ Screen: A tool for multi-genome mapping and quality control. F1000Res 7, 1338.

52. Dobin, A., Davis, C.A., Schlesinger, F., Drenkow, J., Zaleski, C., Jha, S., Batut, P., Chaisson, M., and Gingeras, T.R. (2013). STAR: ultrafast universal RNA-seq aligner. Bioinformatics 29, 15-21.

53. Anders, S., and Huber, W. (2010). Differential expression analysis for sequence count data. Genome Biol 11, R106.

54. Le Meur, N., and Gentleman, R. (2012). Analyzing biological data using R: methods for graphs and networks. Methods Mol Biol 804, 343-373. 
55. Zhou, Y., Zhou, B., Pache, L., Chang, M., Khodabakhshi, A.H., Tanaseichuk, O., Benner, C., and Chanda, S.K. (2019). Metascape provides a biologist-oriented resource for the analysis of systems-level datasets. Nat Commun 10, 1523.

56. Richardson, J.E., and Bult, C.J. (2015). Visual annotation display (VLAD): a tool for finding functional themes in lists of genes. Mamm Genome 26, 567-573.

57. Sekiguchi, R., and Hauser, B. (2019). Preparation of Cells from Embryonic Organs for Single-Cell RNA Sequencing. Curr Protoc Cell Biol 83, e86.

58. Stuart, T., Butler, A., Hoffman, P., Hafemeister, C., Papalexi, E., Mauck, W.M., 3rd, Hao, Y., Stoeckius, M., Smibert, P., and Satija, R. (2019). Comprehensive Integration of SingleCell Data. Cell 177, 1888-1902 e1821.

59. Butler, A., Hoffman, P., Smibert, P., Papalexi, E., and Satija, R. (2018). Integrating singlecell transcriptomic data across different conditions, technologies, and species. Nat Biotechnol 36, 411-420.

60. Butler, A., Hoffman, P., Smibert, P., Papalexi, E., and Satija, R. (2018). Integrating singlecell transcriptomic data across different conditions, technologies, and species. Nature Biotechnology 36, 411-420.

61. Stuart, T., Butler, A., Hoffman, P., Hafemeister, C., Papalexi, E., Mauck, W.M., III, Hao, Y., Stoeckius, M., Smibert, P., and Satija, R. (2019). Comprehensive Integration of SingleCell Data. Cell 177, 1888-1902.e1821.

62. Chung, N.C., and Storey, J.D. (2015). Statistical significance of variables driving systematic variation in high-dimensional data. Bioinformatics 31, 545-554.

63. Macosko, Evan Z., Basu, A., Satija, R., Nemesh, J., Shekhar, K., Goldman, M., Tirosh, I., Bialas, Allison R., Kamitaki, N., Martersteck, Emily M., Trombetta, John J., Weitz, David A., Sanes, Joshua R., Shalek, Alex K., Regev, A., and McCarroll, Steven A. (2015). Highly Parallel Genome-wide Expression Profiling of Individual Cells Using Nanoliter Droplets. Cell 161, 1202-1214.

64. McInnes, L., and Healy, J. (2018). UMAP: Uniform Manifold Approximation and Projection for Dimension Reduction.

65. Du, Y., Guo, M., Whitsett, J.A., and Xu, Y. (2015). 'LungGENS': a web-based tool for mapping single-cell gene expression in the developing lung. Thorax 70, 1092-1094.

66. Zhang, X., Lan, Y., Xu, J., Quan, F., Zhao, E., Deng, C., Luo, T., Xu, L., Liao, G., Yan, M., Ping, Y., Li, F., Shi, A., Bai, J., Zhao, T., Li, X., and Xiao, Y. (2018). CellMarker: a manually curated resource of cell markers in human and mouse. Nucleic Acids Research 47, D721-D728.

67. Haghverdi, L., Lun, A.T.L., Morgan, M.D., and Marioni, J.C. (2018). Batch effects in singlecell RNA-sequencing data are corrected by matching mutual nearest neighbors. Nature Biotechnology 36, 421-427.

68. Beauchemin, K.J., Wells, J.M., Kho, A.T., Philip, V.M., Kamir, D., Kohane, I.S., Graber, J.H., and Bult, C.J. (2016). Temporal dynamics of the developing lung transcriptome in three common inbred strains of laboratory mice reveals multiple stages of postnatal alveolar development. PeerJ 4, e2318.

69. Desai, T.J., Brownfield, D.G., and Krasnow, M.A. (2014). Alveolar progenitor and stem cells in lung development, renewal and cancer. Nature 507, 190-194.

70. Yvernogeau, L., Klaus, A., Maas, J., Morin-Poulard, I., Weijts, B., Schulte-Merker, S., Berezikov, E., Junker, J.P., and Robin, C. (2020). Multispecies RNA tomography reveals regulators of hematopoietic stem cell birth in the embryonic aorta. Blood 136, 831-844.

71. McGowan, S.E., Grossmann, R.E., Kimani, P.W., and Holmes, A.J. (2008). Platelet-derived growth factor receptor-alpha-expressing cells localize to the alveolar entry ring and have characteristics of myofibroblasts during pulmonary alveolar septal formation. Anat Rec (Hoboken) 291, 1649-1661. 
72. Branchfield, K., Li, R., Lungova, V., Verheyden, J.M., McCulley, D., and Sun, X. (2016). A three-dimensional study of alveologenesis in mouse lung. Dev Biol 409, 429-441.

73. Gouveia, L., Betsholtz, C., and Andrae, J. (2018). PDGF-A signaling is required for secondary alveolar septation and controls epithelial proliferation in the developing lung. Development 145.

74. Betsholtz, C. (2004). Insight into the physiological functions of PDGF through genetic studies in mice. Cytokine Growth Factor Rev 15, 215-228.

75. Ntokou, A., Klein, F., Dontireddy, D., Becker, S., Bellusci, S., Richardson, W.D., Szibor, M., Braun, T., Morty, R.E., Seeger, W., Voswinckel, R., and Ahlbrecht, K. (2015). Characterization of the platelet-derived growth factor receptor-alpha-positive cell lineage during murine late lung development. Am J Physiol Lung Cell Mol Physiol 309, L942958.

76. Li, R., Bernau, K., Sandbo, N., Gu, J., Preissl, S., and Sun, X. (2018). Pdgfra marks a cellular lineage with distinct contributions to myofibroblasts in lung maturation and injury response. Elife 7.

77. Naizhen, X., Kido, T., Yokoyama, S., Linnoila, R.I., and Kimura, S. (2019). Spatiotemporal Expression of Three Secretoglobin Proteins, SCGB1A1, SCGB3A1, and SCGB3A2, in Mouse Airway Epithelia. J Histochem Cytochem 67, 453-463.

78. Dixit, R., Ai, X., and Fine, A. (2013). Derivation of lung mesenchymal lineages from the fetal mesothelium requires hedgehog signaling for mesothelial cell entry. Development 140, 4398-4406.

79. Moiseenko, A., Kheirollahi, V., Chao, C.M., Ahmadvand, N., Quantius, J., Wilhelm, J., Herold, S., Ahlbrecht, K., Morty, R.E., Rizvanov, A.A., Minoo, P., El Agha, E., and Bellusci, S. (2017). Origin and characterization of alpha smooth muscle actin-positive cells during murine lung development. Stem Cells 35, 1566-1578.

80. del Moral, P.M., De Langhe, S.P., Sala, F.G., Veltmaat, J.M., Tefft, D., Wang, K., Warburton, D., and Bellusci, S. (2006). Differential role of FGF9 on epithelium and mesenchyme in mouse embryonic lung. Dev Biol 293, 77-89.

81. Yi, L., Domyan, E.T., Lewandoski, M., and Sun, X. (2009). Fibroblast growth factor 9 signaling inhibits airway smooth muscle differentiation in mouse lung. Dev Dyn 238, 123137.

82. Goodwin, K., Mao, S., Guyomar, T., Miller, E., Radisky, D.C., Kosmrlj, A., and Nelson, C.M. (2019). Smooth muscle differentiation shapes domain branches during mouse lung development. Development 146.

83. Danopoulos, S., Shiosaki, J., and Al Alam, D. (2019). FGF Signaling in Lung Development and Disease: Human Versus Mouse. Front Genet 10, 170.

84. Shefer, G., and Benayahu, D. (2010). SVEP1 is a novel marker of activated pre-determined skeletal muscle satellite cells. Stem Cell Rev Rep 6, 42-49.

85. Streuli, C. (1999). Extracellular matrix remodelling and cellular differentiation. Curr Opin Cell Biol 11, 634-640. 
Figure 1

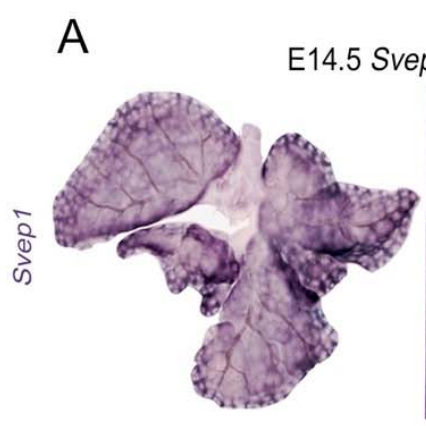

B

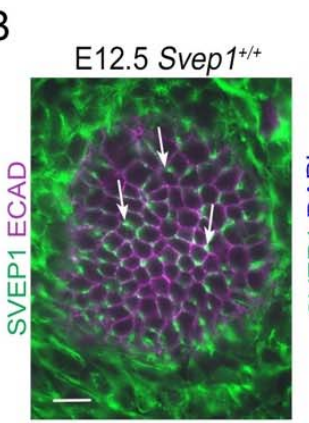

C ${ }_{\text {E14.5 Svep }{ }^{1+*}}$

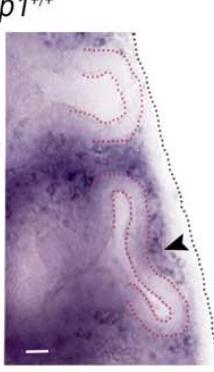

D

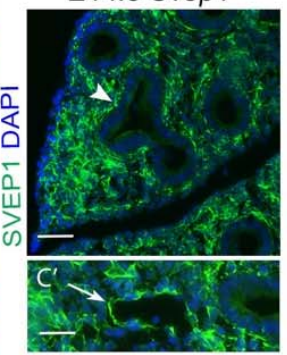

E18.5 Svep $1^{+/+}$

E

E18.5 Svep1 ${ }^{1 /+}$

E18.5 Svep1

F
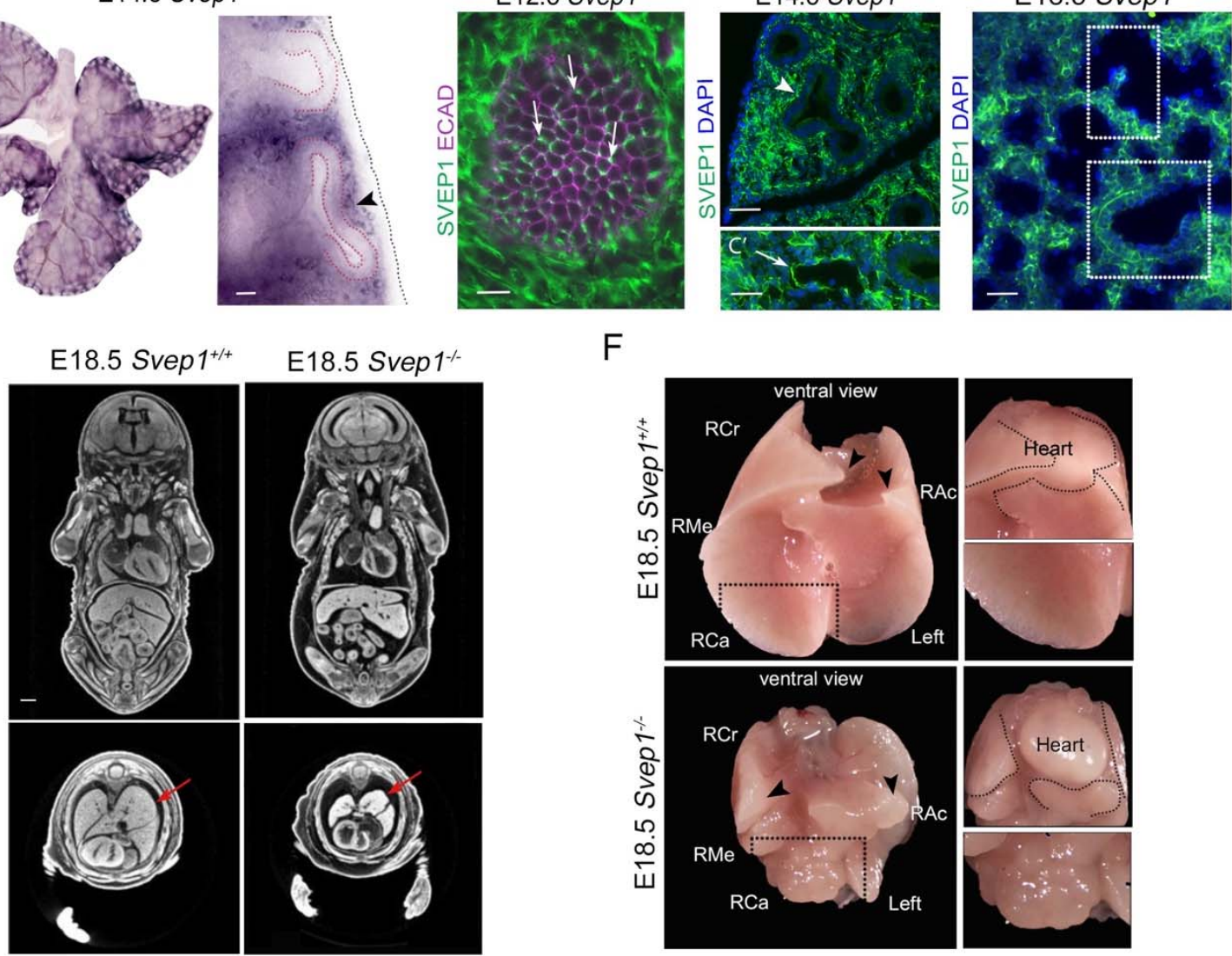

G

H\&E

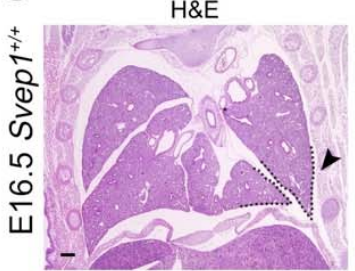

ECAD SOX9 DAPI
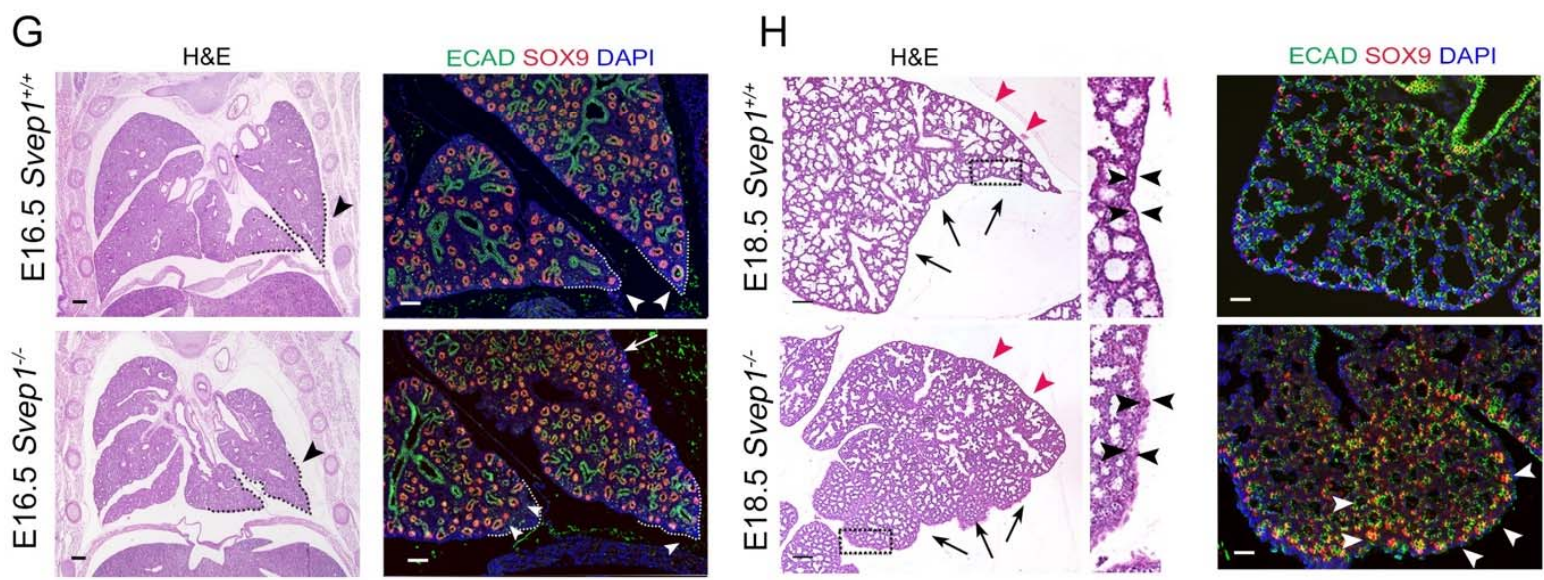

Figure 2 
Figure 1. Lungs of Svep1/- mice are hypoplastic and lobulated. (A) In situ hybridization of E14.5 wild type mice lungs showed strong Svep1 mRNA expression in the mesenchyme at the lung periphery and around bifurcating distal airways tips (right inset outlined in red, arrowhead). (B-D) SVEP1 protein immunofluorescence in the lung mesenchyme of Svep $1^{+/+}$embryos. (B) White arrows indicate the location of the SVEP1 (green) protein between the epithelial membrane stained with ECAD (purple) in the most distal airway buds of the lungs of Svep $1^{+/+}$ E12.5 embryos. (C) The mesenchyme of E14.5 lung tips expresses a high level of SVEP1. The white arrowhead shows branching cleft sites, while the white arrow indicates the growing vasculature. (D) SVEP1 protein in E18.5 lungs is located in the lung parenchyma including the primary septa and adjacent to the proximal epithelium. (E) microCT scan of E18.5 embryos of showing lung hypoplasia (red arrows) and potential thin diaphragm in Svep ${ }^{-}$mice relative to normal controls. (F) Ventral view of E18.5 of Svep $1^{+/+}$and Svep $1^{/-}$lungs. Lung lobes in Svep $1^{-/-}$ mice are rounded and fail to cover the heart (black arrowheads). The right caudal lobe (RCa) of Svep $1^{-/}$embryos has a lobulated appearance that resembles cauliflower (inset). (G) H\&E stained lung sections from E16.5 embryos demonstrating that, compared to wild type embryos, lungs from Svep 1/- embryos are smaller and have irregular lobe edges (black arrowheads). Colocalization of epithelial marker (ECAD) and epithelial progenitor maker (SOX9) showing distal airways that are densely packed in Svep $1^{--}$lungs at E16.5 (white arrows). Distal tips of lungs from mutant embryos are rounded and exhibit an increased density of distal airways (white arrowheads). (H) H\&E stained sections of E18.5 lungs showing the dorsal (red arrowheads) and ventral side (black arrows) of E18.5 lungs. Svep1 ${ }^{/-}$lungs show defects in sacculation, particularly in the lobulated ventral side of the lung (black arrows), and hypercellular mesothelium (arrowhead in inserts). Co-localization of ECAD and SOX9 showing high expression of SOX9 in the lung edge distal airways of Svep $1^{-/}$embryos at E18.5 (white arrowheads), Right cranial (RCr), Right Caudal (RCa), Right Medial (RMe), and Right Accessory (RAc) lobes. Scale bars: $50 \mathrm{~mm}(\boldsymbol{A}, \boldsymbol{B}, \boldsymbol{C}, \boldsymbol{D}), 100 \mathrm{~mm}(\mathbf{G}, \boldsymbol{H})$. 


\section{Figure 2}

A

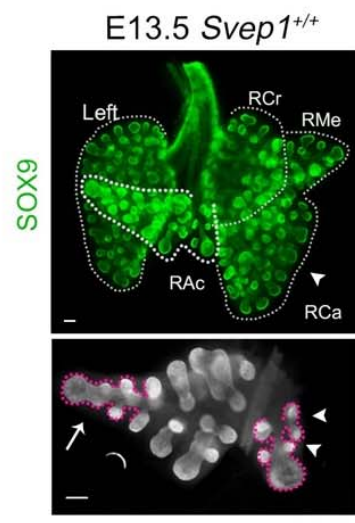

E13.5 Svep1\%

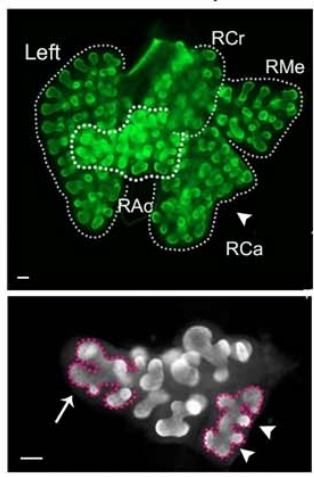

B

E13.5 Svep1+/+

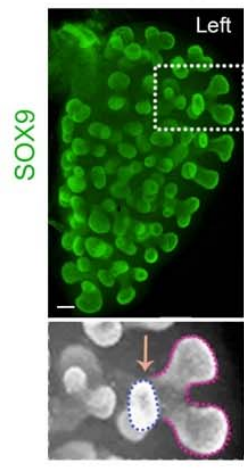

E13.5 Svep1/-

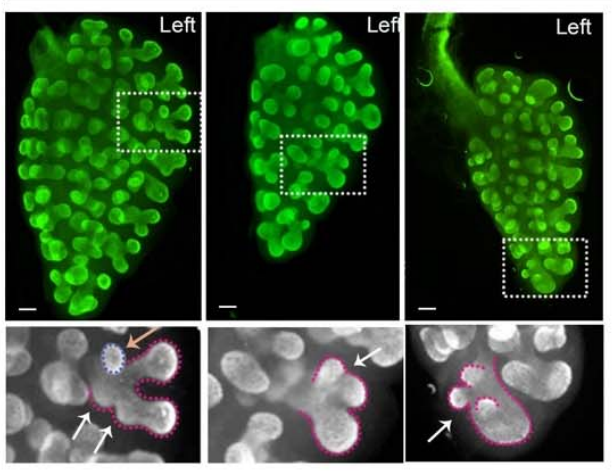

E

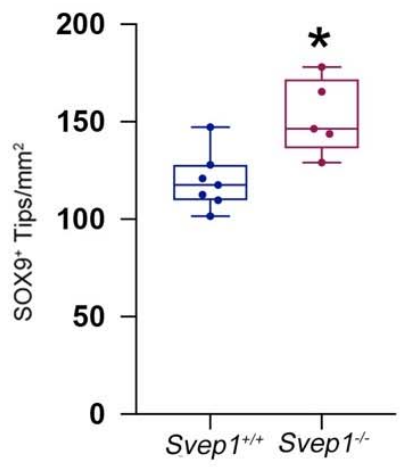

Svep $1^{1 /+}$ Svep $1^{-}$
C

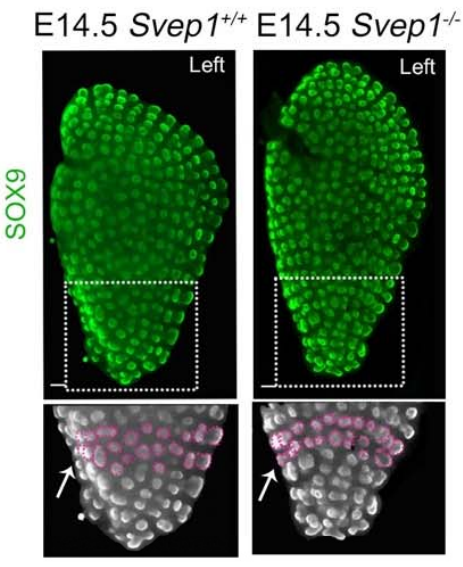

D

E14.5 Svep1+/+
E14.5 Svep1

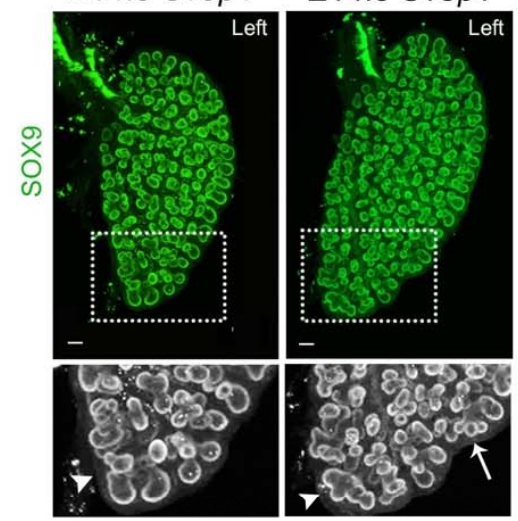


Figure 2. Loss of Svep1 leads to an aberrant increased in distal airways resulting in lobe shape defects. Right cranial (RCr), Right Caudal (RCa), Right Medial (RMe), and Right Accessory (RAc) lobes. Scale bars: 100mm (A-D) Representative Z-stack images showing SOX9 immunofluorescence of whole lung or individual lobes in Svep $1^{+/+}$and Svep $1^{1-}$ mice at E13.5 and E14.5. (A) Whole lungs at E13.5 illustrating the abnormal shape of the RCa lobe in the lung of mutant lung embryos (white arrowhead). Main bronchi of lungs from Svep $1^{+/+}$ embryos end a solo distal tip (lower left inset; white arrow) and two distal tips in Svep $1^{-1}$ embryos (right lower inset; white arrow). Lung budding defects are also observed in mutant embryos (right lower inset; white arrowheads). (B) Left lung lobes of Svep $1^{+/+}$and Svep $1^{-/}$ embryos at E13.5 illustrating branching/budding defects in mutant embryos. The areas outlined in pink in the bottom insets show the main airways. E13.5 Abnormal branching/budding in the distal lobe of Svep 1 - embryos marked by arrows (orange arrow points to a mis localized airway bud). (C) E14.5 left lung lobes of Svep $1^{+/+}$and Svep $1^{\prime-}$ embryos. SOX9 buds highlighted in pink show abnormal airway tip arrangement (white arrows) in mutants resulting in abnormal tip lung tip morphology in Svep 1/ embryos. (D) Immunofluorescence of SOX9 of the left lung lobe at E14.5 shows airway disorganization in Svep $1^{-/}$compared to Svep $1^{+/+}$embryos. Distal lung tips indicated by arrowheads. Branching trifurcation in the Svep $1^{-/}$embryo is shown (white arrow). (E) Lungs of Svep 1/- embryos at E14.5 have a statistically significant increase in number of SOX9 positive airway tips compared to wild type embryos. 
bioRxiv preprint doi: https://doi.org/10.1101/2021.07.26.453586; this version posted July 26, 2021. The copyright holder for this preprint (which was not certified by peer review) is the author/funder. All rights reserved. No reuse allowed without permission.

Figure 3
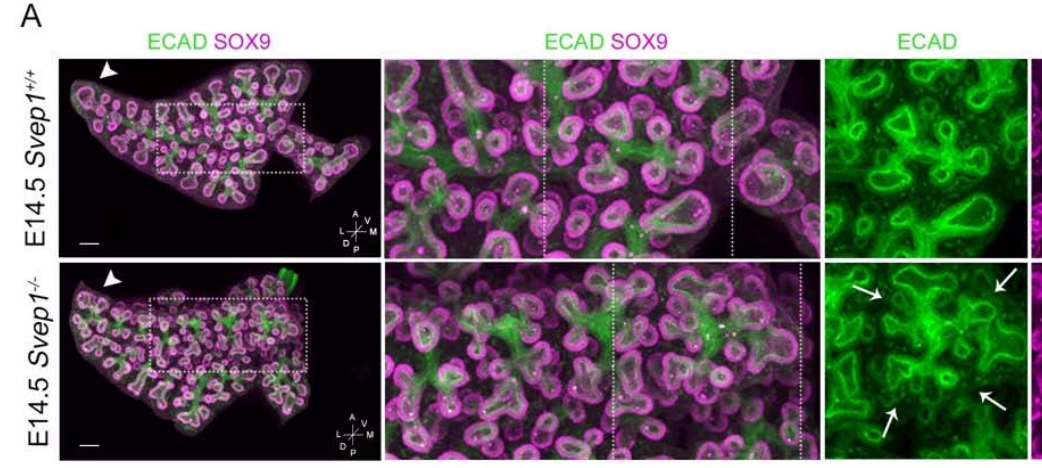

$\operatorname{sox} 9$

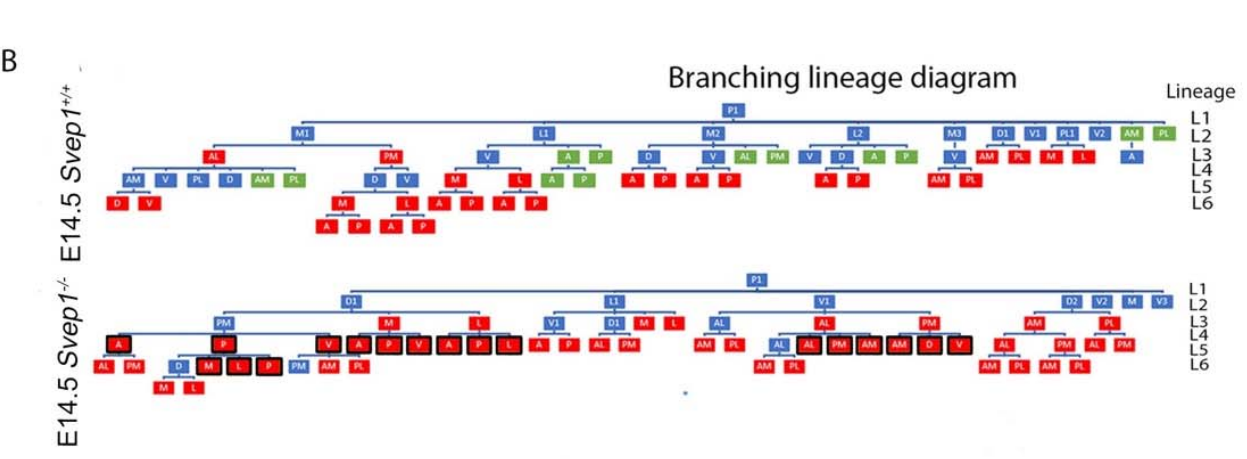

C ECAD SOX9

B

Domain $\mathbf{E}$ Orthogonal $\mathbf{E}$ Planar $\mathbf{\square}$ Trifurcation

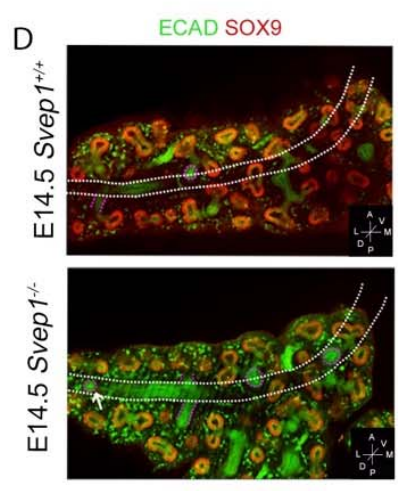

E

E12.5 Svep1 1/+

E12.5 Svep1
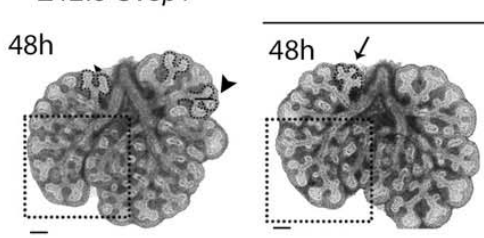

E12.5 Svep1
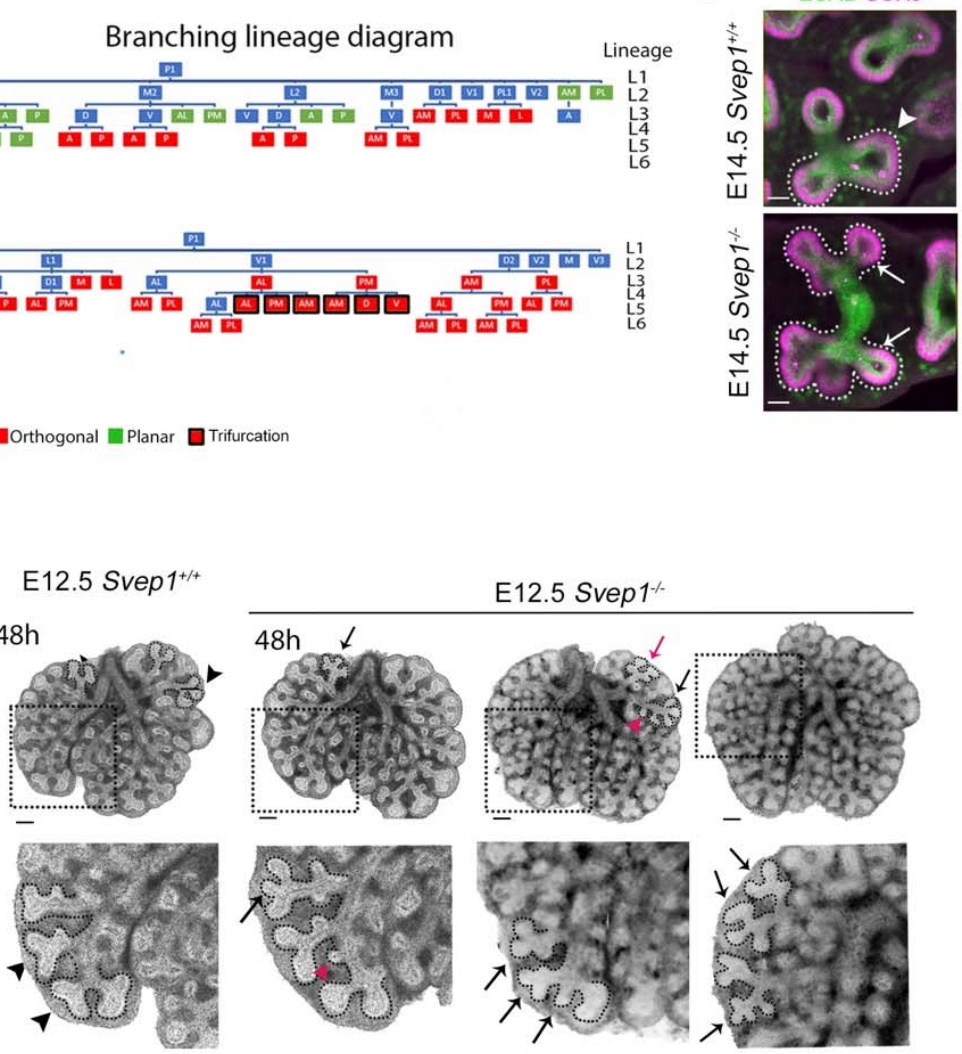
Figure 3. Svep1 ${ }^{-/-}$lung airways undergo ectopic branching and increased trifurcation. (A) Compilation of a Z-stack image series for tissue-cleared whole RAc lung lobes labeled with of ECAD (green) and SOX9 (red) showing disorganized branching in Svep 1/ embryos at E14.5 (highlight). Arrows point to branching airways with rosette-like arrangement and airway tips growing in different directions. Arrowheads indicate the RCa lobe tips showing a single tip in the lobe of Svep $1^{+/+}$embryos and multi branched tips in Svep1 knockout embryos. (B) Schematic illustrating representative domain (blue), planar (green), and orthogonal (red) branching programs of Svep $1^{+/+}$and Svep $1^{-/-}$RAc lobes. Lungs of Svep $1^{-/}$embryos lack planar bifurcations and have many trifurcating branch tips (red highlighted in black). (C) Single Z-stack image of RAc lobes at E14.5 stained with ECAD (green) and SOX9 (red) showing bifurcations (white dotted lines and a white arrowhead) in Svep $1^{+/+}$embryos and trifurcations (white dotted lines and white arrows) in Svep 1/- mice. (D) Single Z-stack image of RAc lobes stained with ECAD (green) and SOX9 (red) displaying ectopic branching in first domain in Svep $1^{-/}$mice. (E) Lung explants from E12.5 embryos after 48 hours in organ culture. Explants from Svep $1^{+/+}$ embryos show normal bifurcation (black arrowheads) whereas explants from Svep $1^{-/}$embryos show obvious trifurcations (black arrows) and ectopic budding (red arrowheads). Scale bars $100 \mu m(A, E) ; 50 \mu m(C)$ 


\section{Figure 4}
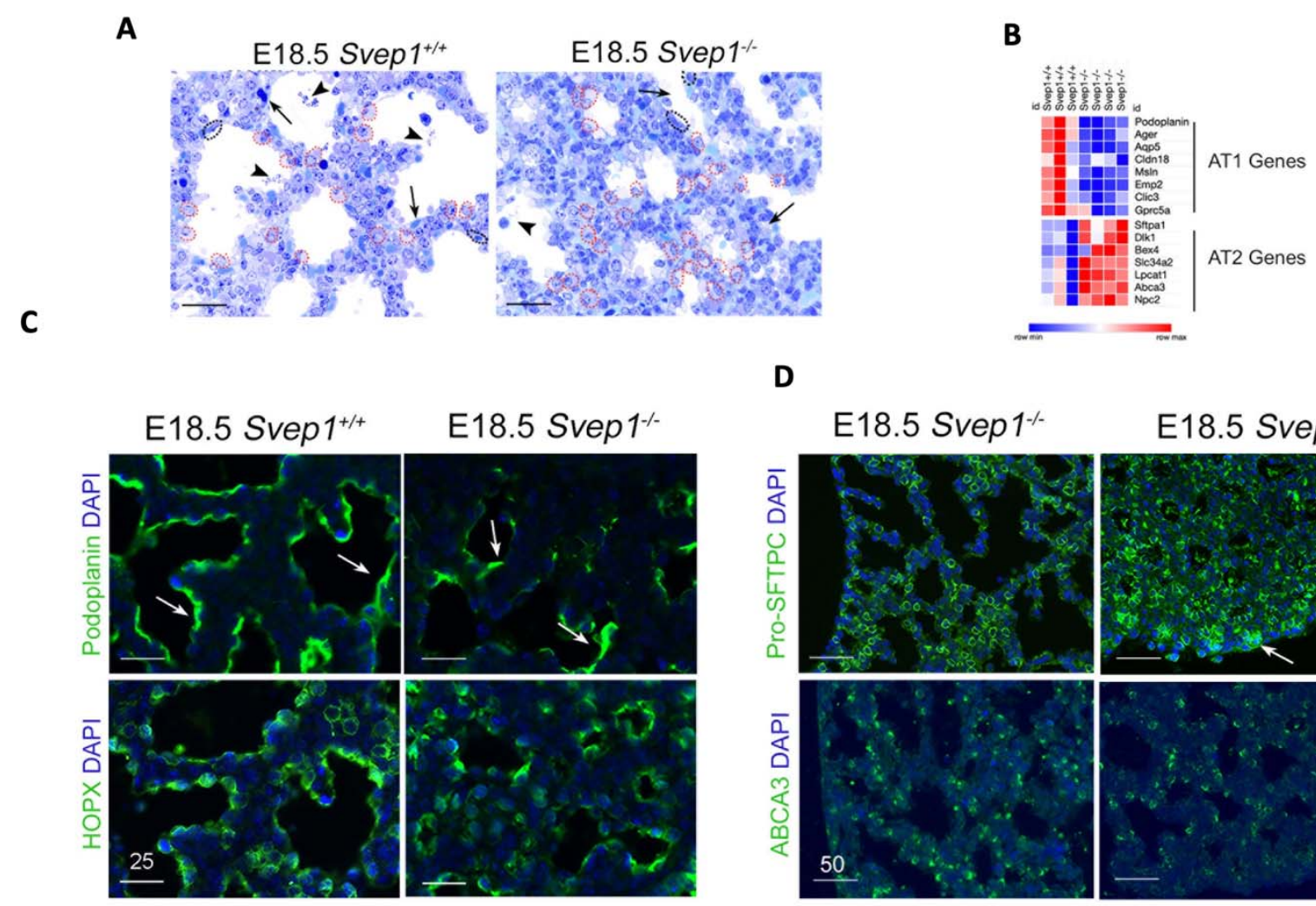

D

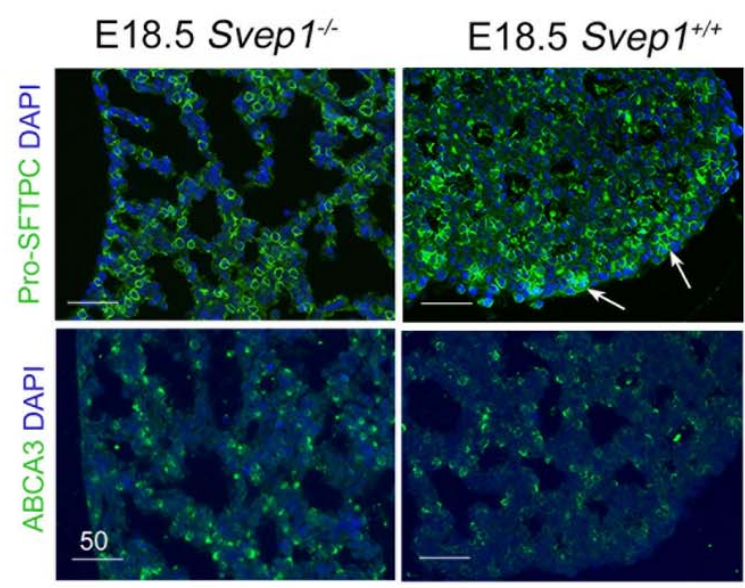

Figure 4. Svep1 mutants have deficiencies in alveolar differentiation. (A) Paraffinembedded semi-thin section of E18.5 lungs stained with toluidine blue. Type I pneumocytes (AT1) are outlined by black dotted lines; Type II pneumocytes (AT2) are outlined with red dotted lines. Round AT2 clusters tightly in immature saccules in Svep ${ }^{1 /}$ lungs. Black arrowheads indicate secreted surfactant protein; black arrows indicate capillaries. (B) Expression heatmaps of selected genes associated with type I (AT1) and type II pneumocytes (AT2). Svep $1^{\text {-1 lungs }}$ have lower expression of type 1 genes compared to Svep $1^{+/+}$lungs and higher expression of type 2 associated genes. (C) Immunofluorescence of Podoplanin (arrows) in the lungs of E18.5 embryos. (D) Localization of Pro-SFTPC in E18.5 lungs. Arrowheads indicate Pro-SFTPC in the lung edges distal epithelium. Scale bars $25 \mu m(F) ; 50 \mu m(G)$ 


\section{Figure 5}

A

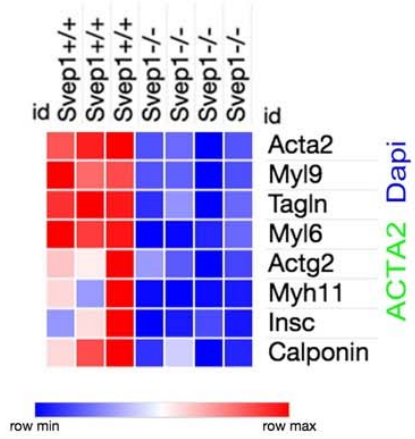

B

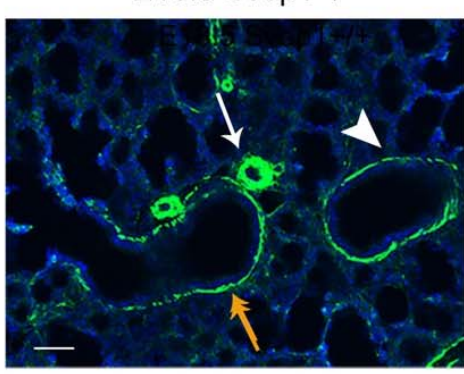

E18.5 Svep1-/-

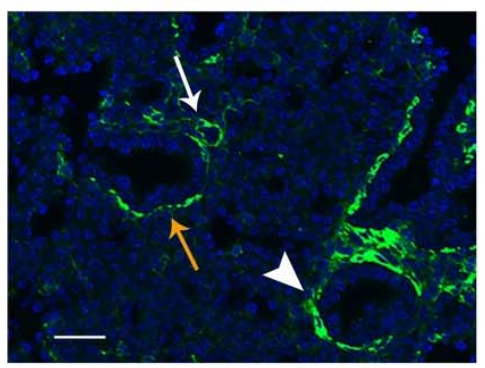

C E12.5 Svep1+/+ E12.5 Svep1-/-
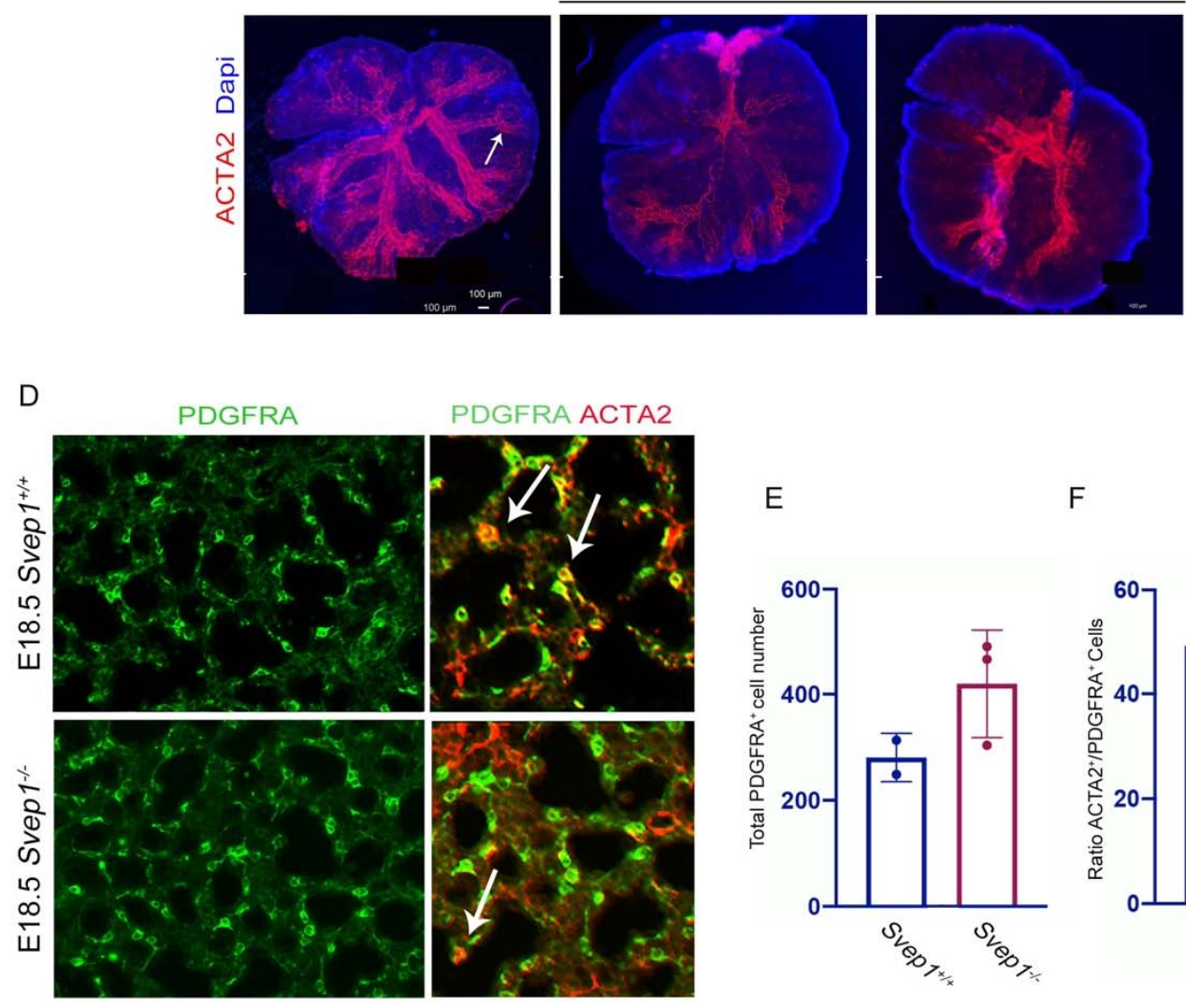

$\mathrm{E}$

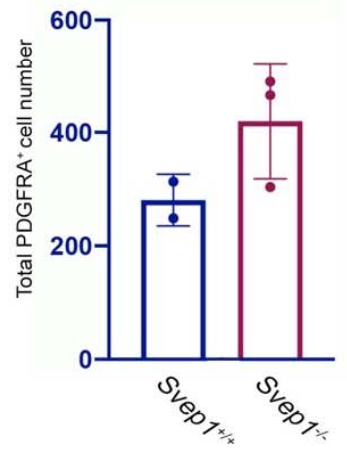

$\mathrm{F}$

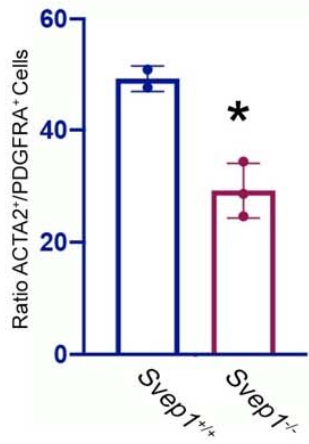


Figure 5. Svep1 ${ }^{-/}$embryos have impaired smooth differentiation in the distal lung. (A) Gene expression heatmap from bulk RNA Seq data showing the top downregulated genes in lungs from Svep 1\% embryos associated with smooth muscle cells. (B) ACTA2 localization marking smooth muscle cells at E18.5. White arrows indicate respiratory bronchioles; orange arrows indicate proximal bronchioles; white arrowheads indicate blood vessels (C) ACTA2 staining (red) in wild type and Svep $1^{-/}$E12.5 lung explants that were cultured for six days show a dramatic reduction of smooth muscle cell formation in secondary and tertiary bronchi (white arrow). (D) Alveolar localization of PDGFRA (green) labeled progenitors and ACTA2 (red) labeled mature myofibroblast (arrows). (E-F) Plots show PDGFRA total count (E) and the ratio of ACTA2+ cells that are also PDGRFA+ $(F)$. Data shown are average counts $\pm S D ; p<0.05$. 


\section{Figure 6}

A

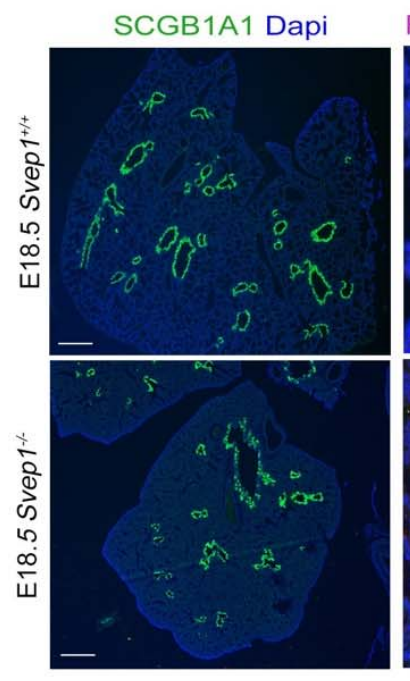

C
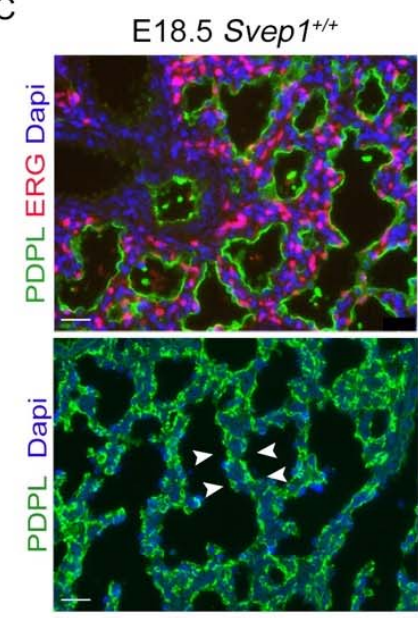

E
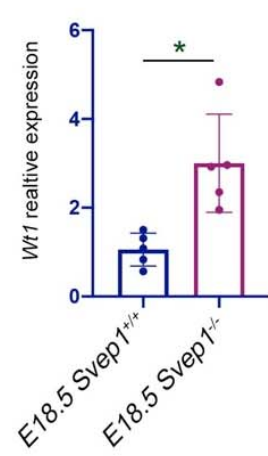

FOXJ1 SCGB1A1 Dapi

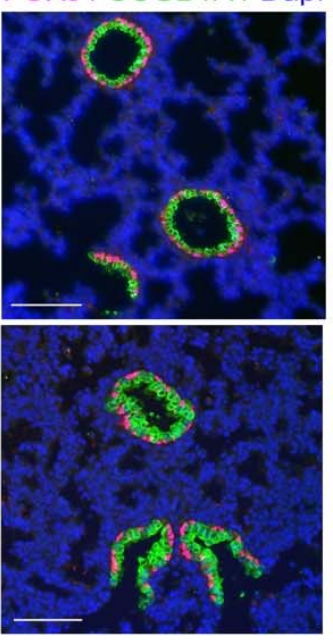

E18.5 Svep 1\%
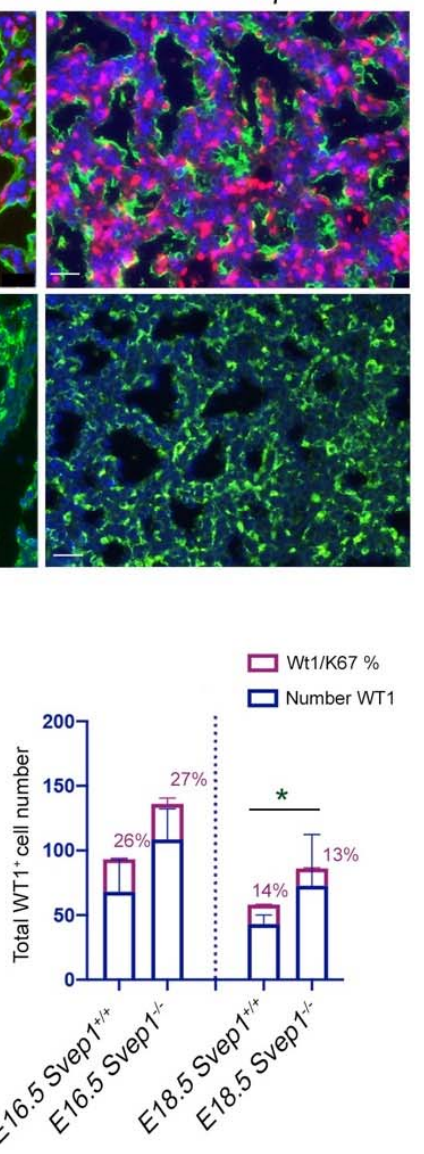

B
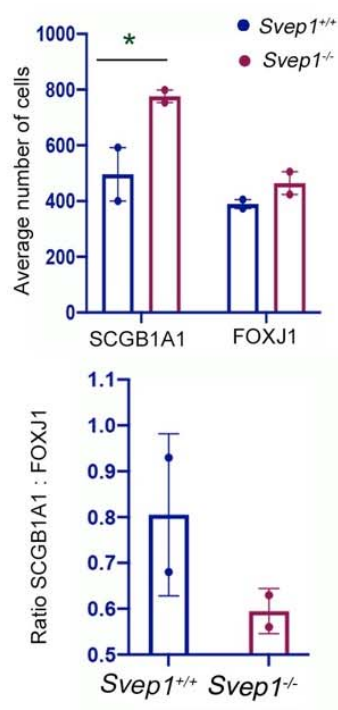

D

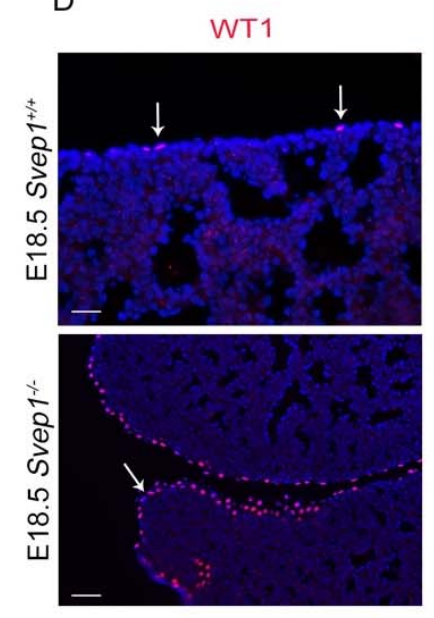

$\mathrm{F}$

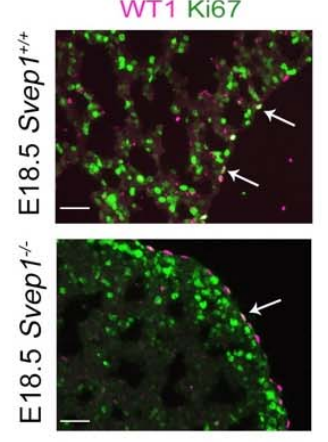


Figure 6. Loss of Svep1 leads to proximal conductive airway hypoplasia and defects in microvasculature formation and mesothelium differentiation. (A) Immunofluorescence of SCGB1A1 (green) club cell marker highlights the lung proximal airways which are reduced in mutants. SCGB1A1 co-localization with the ciliated cell marker FOXJ1 (purple) reveals a multicellular epithelium constituted primarily of club cells. In Svep $1^{-/}$embryos, the lumen of bronchioles is narrowed. (B) Plots showing quantification of SCGB1A1+ and FOXJ1+ cells and ratios in Svep $1^{-/}$and Svep $1^{+/+}$embryos. (C) Co-labeling of endothelial cell nucleus with ERG (red) and type I pneumocytes (AT1) with Podoplanin (PDPL; green) at E18.5. (D) Immunofluorescence for the mesothelial progenitor marker WT1 (red) showing that Svep $1^{-}$ lungs have a greater number of WT1 progenitors. (E) Plots exhibit the total number of WT1 at E18.5 and percentage of WT1+ and KI67+ at E16.5 and A18.5. (F) Immunofluorescence for WT1 (purple) and KI67 (green). Arrows indicate regions of WT1+ cell proliferation. Scale bars $200 \mu m(D) ; 100 \mu m(B, D, E, G)$. 


\section{Figure 7}

A
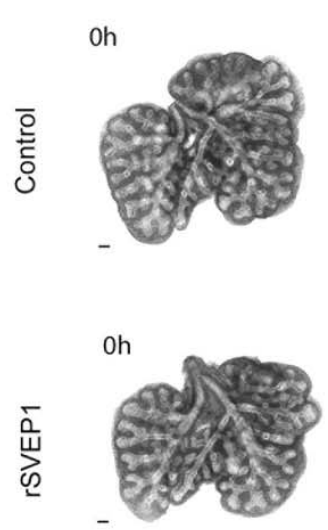

$24 \mathrm{~h}$

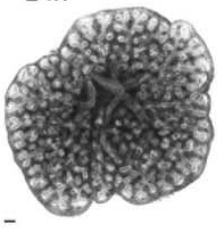

$24 \mathrm{~h}$

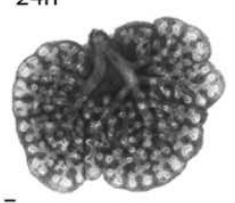

$48 \mathrm{~h}$

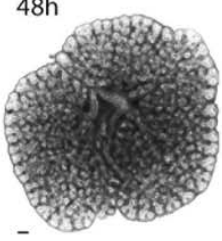

$48 \mathrm{~h}$

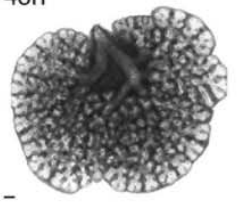

B

\section{Peripheral airway counts}

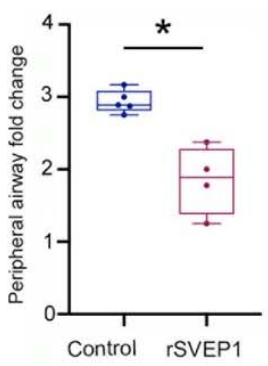

C

E12.5 Svep1 $1^{1 /+}$

E12.5 Svep1\%, SVEP1 peptide

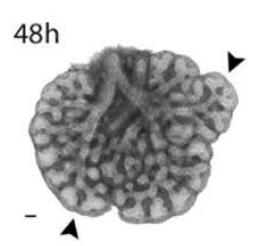

D
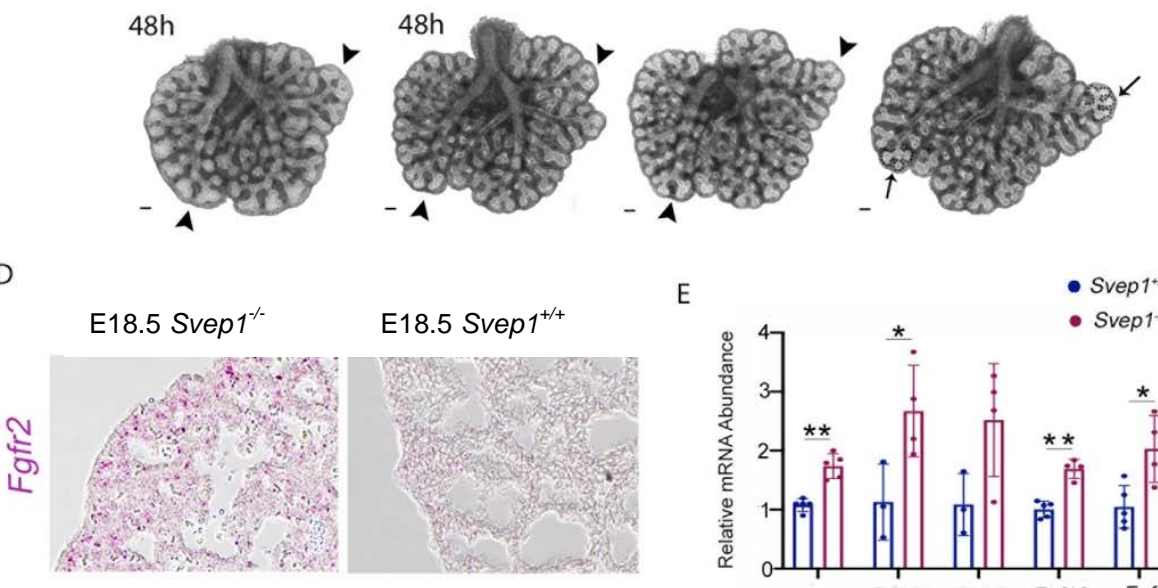

E
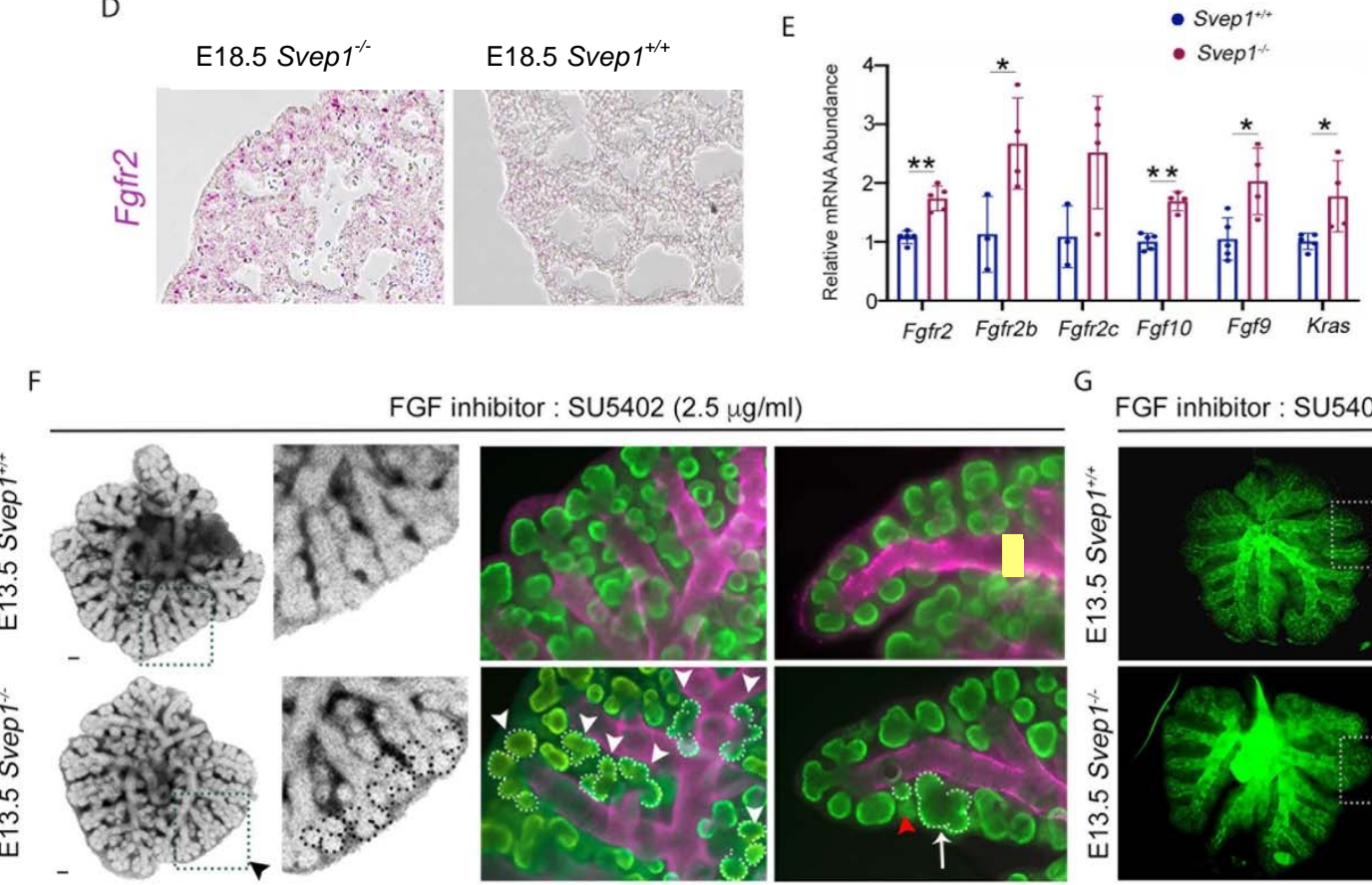

FGF inhibitor : SU5402 $(2.5 \mu \mathrm{g} / \mathrm{ml})$
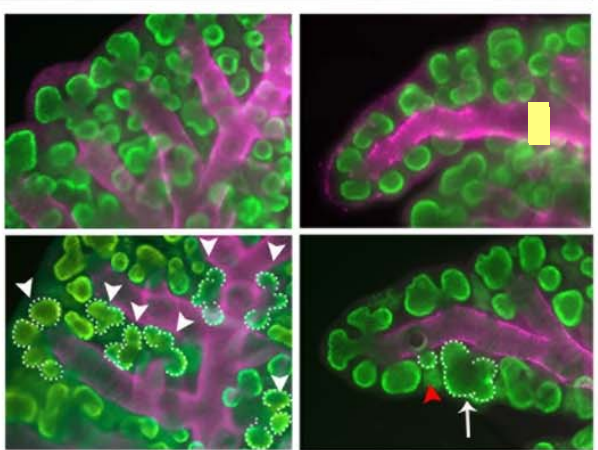

SOX9ACTA2

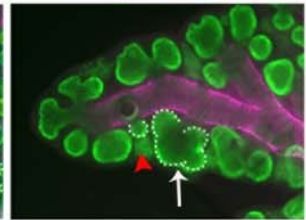

SOX9 ACTA2
G

FGF inhibitor : SU5402 $(2.5 \mu \mathrm{g} / \mathrm{ml})$

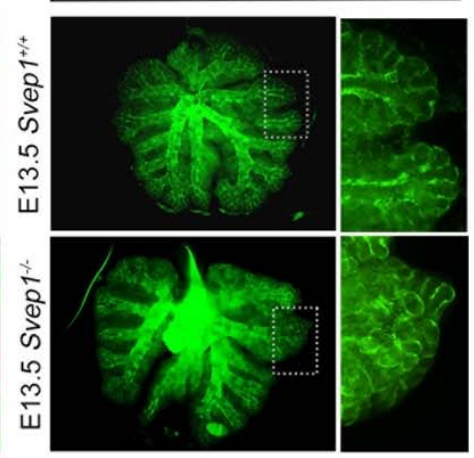

ACTA2 
Figure 7. SVEP1 acts as a negative regulator of branching and the FGF pathway mediates smooth muscle differentiation defects in Svep1 ${ }^{-/}$lungs. (A) Lung explants from E12.5 Svep $1^{+/+}$embryos cultured for 48 hours with and without SVEP1 peptide. Treated lung explants show a reduction of branching. (B) Plot of peripheral airways counts in lung explants from Svep $1^{+/+}$and Svep $1^{-/}$E12.5 embryos following SVEP1 peptide treatment. Svep $1^{-/}$lung explants show a significant reduction of peripheral airways. (C) E12.5 lung explants treated with SVEP1 peptide ex vivo for 48 hours. Black arrowheads indicate normal bifurcation in $S v e p 1^{+/+}$and Svep $1^{-/}$lungs. Arrows indicate rare trifurcations in Svep $1^{-/}$lung explants treated with rSVEP1. (D) In situ hybridization of Fgfr2 demonstrates increased expression in Svep 1/ mutants. (E) Plot of relative transcript abundance of Fgfr2 (and its isoforms Fgf2b and Fgfr2c), Fgf10, Fgf9, and Kras. (F) Ex vivo Svep $1^{+/+}$and Svep $1^{-/}$lungs from E12.5 treated with the FGF signaling inhibitor SU5402. The regions outlined with dotted lines are shown in higher magnification and demonstrate the distal tip trifurcations in lung explants from Svep $1^{-/}$embryos. SOX9 (green) and ACTA2 (purple) staining confirm branching anomalies (highlighted in the white dotted areas) in Svep $1^{-/}$lung explants. Arrowheads point to trifurcations; arrows indicate abnormal branching; the red arrowhead highlights ectopic budding. (G) E12.5 lung explants treated with SU5402 $(2.5 \mu \mathrm{g} / \mathrm{ml})$ for 4 days stained with ACTA2 (green). Inserts showing ACTA2 localization in distal airways of normal and mutant lungs. 